\title{
Effect of fiber-orientation on stresses at the supporting end of a short cantilever beam of fiber-reinforced composites
}

\author{
Md Jamil Hossain \\ Department of Mechanical Engineering, \\ Bangladesh University of Engineering \& Technology, Dhaka 1000, Bangladesh
}

\begin{abstract}
The effect of fiber orientation on the state of stress, especially around the supporting end of a short cantilever beam of fiber-reinforced composites is investigated. Two limiting cases of fiber orientation are considered for the composite beam. A displacement potential based finitedifference computational scheme is used to obtain the numerical solution of the elastic field of the problem. Analysis of the results shows that the fiber orientation plays an important role in defining the state of stresses, especially around the supporting end of a cantilever beam. The fiber orientation effect is also identified to be influenced by a number of practical issues like, beam aspect-ratio, supporting condition, type of loading and fiber stiffness. Finally, a comparative analysis of the results with the corresponding FEM solution is included to verify the appropriateness as well as accuracy of the present computational study.
\end{abstract}

\section{Keywords}

Stress analysis, fiber-reinforced composite, cantilever beam, displacement potential, finite difference method, finite element method. 


\section{Introduction}

Composite materials are now-a-days extensively used in industries, especially in the construction of aerospace and marine structures as well as machine elements. This is mainly because of their improved characteristics and properties compared to those of conventional isotropic materials. It is well known that the mechanical properties like, specific strength, stiffness, toughness of a fiber reinforced composite material differ significantly from those of conventional materials, which are however found to depend on how the fibers are oriented within the matrix material. As a result, the fiber orientation plays a dominant role in defining the overall state of stresses and deformation of composite structures under loading. Therefore, a reliable analysis of the effect of fiber orientation is of utmost importance for accurate prediction of mechanical behavior as well as improved, economic design of composite structures.

A cantilever beam is one of the most commonly used structural elements in practical applications. However, exact analytical solution to this classical problem, especially a short one, with complete satisfaction of all the physical conditions imposed on it is still hardly available and thus, is being constantly looked into. Taking the secondary effects into consideration, Rehfield and Murty ${ }^{1}$ derived a bending theory for beams, in which the elasticity

solution of a uniformly loaded simply supported beam, given by Goodier and Timoshenko ${ }^{2}$ was taken as the basis. By including the coupled transverse shear and non-classical axial stress effects Murty ${ }^{3}$ extended his higher order theory to analyze a short cantilever beam with tip load. In this formulation, the strain energy due to lateral stress component was neglected and Poisson's ratio of the beam material was assumed to be zero. His results showed that the shearing stresses along the upper and lower edges do not vanish and their values become large in the neighborhood of fixed edge. Besides the analytical solutions of deep beams, a number 
of numerical investigations have also been reported in the literature. Based on stress function formulation, ${ }^{2}$ simply-supported deep beams were analyzed with different loading conditions by Chow et al. ${ }^{4}$ to predict the bending and shearing stress distributions at different transverse sections of the beams using finite-difference method (FDM). Introducing a boundary modeling approach for FDM applications of displacement formulation of solid mechanics Dow et al. ${ }^{5}$ solved the problem of a uniformly loaded short cantilever beam and verified the superiority of FDM in reproducing the state of stresses at the surfaces. Later, a careful finite-element analysis of short beams with various boundary conditions was conducted by Hardy and Pipelzadeh. ${ }^{6} \mathrm{~A}$ built-in short beam with uniform loading was analyzed for predicting the elastic field by Ahmed et al., ${ }^{7}$ in which a displacement potential based elasticity formulation was used in conjunction with FDM. The computational scheme was further extended to investigate the stresses at the fixed end of short cantilever beams subjected to a shear loading, ${ }^{8}$ which however identified the supporting end of a short cantilever beam to be the most critical section in terms of stresses.

The investigation of the effect of fiber orientation on the mechanical behavior of structural components has now become a key subject in the field of composite structures, and the corresponding reporting in the literature is quite extensive, some of which are cited as examples in References. ${ }^{9-14}$ For practical cases, the investigation is usually found to carry out by numerical approaches, as the exact analytical methods are limited to very ideal cases. Recently, some experimental investigations of the same are also being carried out mainly to validate the theoretical predictions. However, a reliable and accurate investigation of the effect of fiber orientation on the critical stresses, especially in a short cantilever beam of fiber-reinforced composites has not been yet conducted.

Recent research and developments in using the displacement-potential approach have 
generated renewed interest in the field of both analytical and numerical solutions of stress problems of solid mechanics. ${ }^{7,8,15,16}$ Quite recently, Ahmed et al. ${ }^{17}$ proposed the displacementpotential formulation for the solution of general anisotropic composite structures. Later, the use of the formulation to problems of orthotropic composite materials has been extended to obtain analytical and numerical solutions of composite structures and laminates under various

types of loading. ${ }^{18-24}$ In the displacement-potential method, the mixed boundary-value elastic problem is formulated in terms of a potential function of space variables, defined in terms of the displacement components of plane elasticity, which has to satisfy a single partialdifferential equation of equilibrium, and the corresponding numerical solutions are obtained by the use of finite-difference technique.

The present paper describes a new investigation of the effect of fiber orientation on the state of stresses at the supporting end of a short cantilever beam of fiber-reinforced composites using displacement-potential method. Two limiting cases of fiber orientation $\left(\theta=0^{\circ}\right.$ and $\left.90^{\circ}\right)$ are considered for the composite beam. Two different types of supporting as well as loading conditions are considered for the cantilever to investigate their influence on the fiberorientation effect. The results of the present investigation are also analyzed in the perspective of beam aspect ratio as well as fiber stiffness. Finally, some of the results are compared with the corresponding finite element solutions with a view to verify the appropriateness as well as accuracy of the present computational investigation.

\section{Displacement Potential formulation}

For the present beam, the thickness is considered small in comparison with its lateral and longitudinal dimensions. Thus, the problem predominantly falls under the plane stress condition. ${ }^{25}$ With reference to a rectangular coordinate system $(x, y)$, in absence of body forces, 
the two differential equations of equilibrium for orthotropic elastic bodies under the plane stress condition are as follows ${ }^{10,26}$

$$
\begin{aligned}
& \left(\frac{E_{1}^{2}}{E_{1}-v_{12}^{2} E_{2}}\right) \frac{\partial^{2} u_{x}}{\partial x^{2}}+\left(\frac{v_{12} E_{1} E_{2}}{E_{1}-v_{12}^{2} E_{2}}+G_{12}\right) \frac{\partial^{2} u_{y}}{\partial x \partial y}+G_{12} \frac{\partial^{2} u_{x}}{\partial y^{2}}=0 \\
& \left(\frac{E_{1} E_{2}}{E_{1}-v_{12}^{2} E_{2}}\right) \frac{\partial^{2} u_{y}}{\partial y^{2}}+\left(\frac{v_{12} E_{1} E_{2}}{E_{1}-v_{12}^{2} E_{2}}+G_{12}\right) \frac{\partial^{2} u_{x}}{\partial x \partial y}+G_{12} \frac{\partial^{2} u_{y}}{\partial x^{2}}=0
\end{aligned}
$$

Where $u_{x}$ and $u_{y}$ are displacement components in the $x$ and $y$-directions, respectively; $E_{1}$ and $E_{2}$ are the longitudinal and transverse Young's moduli; $v_{12}$ and $v_{21}$ are the major and minor Poisson's ratio and $G_{12}$ is the in-plane shear modulus. The corresponding stress components are related to the displacement components through the following relations ${ }^{26}$

$$
\begin{aligned}
& \sigma_{x x}=\frac{E_{1}}{1-v_{12} v_{21}}\left[\frac{\partial u_{x}}{\partial x}+v_{21} \frac{\partial u_{y}}{\partial y}\right] \\
& \sigma_{y y}=\frac{E_{2}}{1-v_{12} v_{21}}\left[\frac{\partial u_{y}}{\partial y}+v_{12} \frac{\partial u_{x}}{\partial x}\right] \\
& \sigma_{x y}=G_{12}\left[\frac{\partial u_{x}}{\partial y}+\frac{\partial u_{y}}{\partial x}\right]
\end{aligned}
$$

Although the two differential equations of equilibrium are theoretically sufficient to solve the mixed boundary-value elastic problems of orthotropic composite materials, but, in reality, it is extremely difficult to solve for two functions simultaneously satisfying the two second-order elliptic partial differential equations. In order to overcome the difficulty, the existence of a potential function of space variables is investigated in an attempt to reduce the problem to the 
determination of a single variable from a single differential equation of equilibrium. In the present formulation, a potential function $\psi(x, y)$ is thus defined in terms of the two displacement components as. ${ }^{17}$

$$
\begin{aligned}
& u_{x}(x, y)=\frac{\partial^{2} \psi}{\partial x \partial y} \\
& u_{y}(x, y)=-\frac{1}{Z_{1}}\left[\left\{W E_{1}+(1-W) E_{2}\right\} E_{1} \frac{\partial^{2} \psi}{\partial x^{2}}+G_{12}\left(E_{1}-v_{12}^{2} E_{2}\right) \frac{\partial^{2} \psi}{\partial y^{2}}\right]
\end{aligned}
$$

Where $Z_{1}=v_{12} E_{1} E_{2}+G_{12}\left(E_{1}-v_{12}^{2} E_{2}\right)$. The coefficient $W$ is introduced here to take care of the effect of fiber-orientation $(\theta)$ as follows:

$$
W= \begin{cases}1 & \left(\text { for } \theta=0^{\circ}\right) \\ 0 & \left(\text { for } \theta=90^{\circ}\right)\end{cases}
$$

With the above definition of $\psi(x, y)$, the first equilibrium equation (1) is automatically satisfied. Therefore, $\psi$ has to satisfy the second equilibrium equation (2) only. Expressing equation (2) in terms of the potential function $\psi$, the condition that $\psi$ has to satisfy is

$$
\left\{W E_{1}+(1-W) E_{2}\right\} G_{12} \frac{\partial^{4} \psi}{\partial x^{4}}+E_{2}\left(E_{1}-2 v_{12} G_{12}\right) \frac{\partial^{4} \psi}{\partial x^{2} \partial y^{2}}+\left\{(1-W) E_{1}+W E_{2}\right\} G_{12} \frac{\partial^{4} \psi}{\partial y^{4}}=0
$$

Combining equations (2) and (3), the components of stress can be expressed in terms of the potential function, $\psi$ as 


$$
\begin{aligned}
& \sigma_{x x}(x, y)=\frac{E_{1} G_{12}}{Z_{1}}\left[\left\{W E_{1}+(1-W) E_{2}\right\} \frac{\partial^{3} \psi}{\partial x^{2} \partial y}+v_{12} E_{2} \frac{\partial^{3} \psi}{\partial y^{3}}\right] \\
& \sigma_{y y}(x, y)=\frac{E_{1}}{Z_{1}}\left[\left(v_{12} E_{2} G_{12}-E_{1} E_{2}\right) \frac{\partial^{3} \psi}{\partial x^{2} \partial y}-\left\{(1-W) E_{1}+W E_{2}\right\} G_{12} \frac{\partial^{3} \psi}{\partial y^{3}}\right] \\
& \sigma_{x y}(x, y)=\frac{E_{1} G_{12}}{Z_{1}}\left[-\left\{W E_{1}+(1-W) E_{2}\right\} \frac{\partial^{3} \psi}{\partial x^{3}}+v_{12} E_{2} \frac{\partial^{3} \psi}{\partial x \partial y^{2}}\right]
\end{aligned}
$$

Under the present formulation, the problem can now be formulated in such a way that a single function $\psi$ has to be evaluated from the governing equation (4), satisfying the boundary conditions that are specified at the edges of the beam.

\section{Numerical solution}

The essential feature of the present numerical investigation is to get the solution of the governing equation (4) corresponding to both $\theta=0^{\circ}$ and $90^{\circ}$, subject to the appropriate boundary conditions prescribed over the edges of the beam. As far as the solution through the proposed $\psi$-formulation is concerned, attention may be drawn to the following points.

\section{Method of solution}

The limitation and complexity associated with analytical solution ultimately gives rise to the fact that the numerical solution of the present mixed boundary value problem is the only plausible approach. The finite difference technique is used to discretize the governing partial differential equation and also the differential equations associated with the boundary conditions. The discrete values of the potential function $\psi(x, y)$, at mesh points of the domain concerned (Figure 1) is solved from the system of linear algebraic equations resulting from the discretization of the governing equation and the associated boundary conditions. 


\section{Discretization of the domain}

Considering the rectangular shape of the boundary and also the nature of the differential equations involved, rectangular grid points are used all over the region concerned for numerical computation. The overall error of the present computational approach has been kept of the order of $h^{2}$. The governing differential equation, which is used to evaluate the function $\psi(x, y)$ only at the internal mesh points, is expressed in its corresponding difference form using central difference operators, as follows:

$$
\begin{aligned}
& F_{1}\{\psi(i-2, j)+\psi(i+2, j)\}-F_{2}\{\psi(i-1, j)+\psi(i+1, j)\}-F_{3}\{\psi(i, j+1)+\psi(i, j-1)\} \\
& +F_{4} \psi(i, j)+F_{5}\{\psi(i+1, j+1)+\psi(i+1, j-1)+\psi(i-1, j+1)+\psi(i-1, j-1)\} \\
& +F_{6}\{\psi(i, j-2)+\psi(i, j+2)\}=0
\end{aligned}
$$

where,

$$
\begin{aligned}
& F_{1}=P(k / h)^{4}, F_{2}=4 P(k / h)^{4}+2 Q(k / h)^{2}, F_{3}=2 Q(k / h)^{2}+4 R, \\
& F_{4}=6 P(k / h)^{4}+4 Q(k / h)^{2}+6 R, F_{5}=Q(k / h)^{2}, F_{6}=R, P=\left\{W E_{1}+(1-W) E_{2}\right\} G_{12}, \\
& Q=E_{2}\left(E_{1}-2 v_{12} G_{12}\right), \quad R=\left\{(1-W) E_{1}+W E_{2}\right\} G_{12}
\end{aligned}
$$

$h, k$ are the mesh lengths in $x$-and $y$-directions, respectively.

As far as the present central-difference grid structure of the governing equation is concerned, when the point of application $(i, j)$ becomes an immediate neighbor to the physical boundary, it will involve mesh points both interior and exterior to the physical boundary of the beam. Thus, an imaginary boundary, exterior to the physical boundary is introduced so that the application of the governing equation (6), especially to the points in the immediate neighborhood of the physical boundary, involves no points exterior to the imaginary boundary, thereby causing no difficulties in developing a straight forward program using a single version of the governing equation. 
As the differential equations associated with the boundary conditions contain second and third order derivatives of the function $\psi$, the use of central-difference expressions is not practical as, most of the time, it leads to the inclusion of points exterior to the imaginary boundary. The derivatives of the boundary expressions are thus replaced by their corresponding backward or forward difference formula, keeping the order of local truncation error the same, $\mathrm{O}\left(h^{2}\right)$. In order to avoid the inclusion of points exterior to the imaginary boundary, four different sets of finite difference expressions for each of the boundary conditions are developed for the points at different regions of the boundary. These four sets of algebraic equations are derived by adopting different combinations of forward, backward and central difference schemes. The choice of the appropriate set of expressions for a point on the boundary is determined by its position on the boundary, which will avoid the occurrence of additional mesh points external to the imaginary boundary.

\section{Specification of the boundary conditions}

As each mesh point on the physical boundary of the beam entertains two conditions at a time, one is used to evaluate $\psi$ at the nodal point on the physical boundary and the other at the corresponding points on the exterior imaginary boundary. The governing equation is used to evaluate $\psi$ only at the interior mesh points of the plane body. Special cares have been taken to model the boundary conditions associated with the corner points of the geometry, which are, in general, the points of singularity. In the present modeling scheme, three out of the four boundary conditions associated with each corner points are satisfied and the remaining one is treated as redundant. It can be mentioned here that, in case of conventional computational approaches, a maximum of two conditions are taken into account to model the corner nodes.

\section{Evaluation of displacement and stress components}

The finite-difference algebraic equations resulting from the discretization of the governing 
equation as well as the boundary conditions are solved by the use of a direct method of solution. Finally, the same difference equations used for the boundary conditions are again organized for the evaluation of all the parameters of interest in the solution of the cantilever beam from the $\psi$ values at different points of the body, as all the components of stress and displacement are expressed as a summation of different derivatives of the function $\psi$.

\section{Description of the problem}

\section{Geometry of the problem and loading conditions}

The model of a cantilever beam of fiber reinforced composite material subjected to a parabolic end shear loading is shown with reference to a Cartesian coordinate system $x$-y in Figure 2. The origin of the coordinate system is taken at the upper left corner of the beam. The thickness of the beam is assumed to be small compared to its length $(L)$ and its depth $(D)$. The analysis is carried out for two extreme cases of fiber orientation, $\theta=0^{\circ}$ and $90^{\circ} . \theta=0^{\circ}$ and $\theta=90^{\circ}$ represent the cases where the fibers are oriented along the $x$-axis and $y$-axis of the beam, respectively (See Figures 2(a) and (b)). Results are obtained for a number of beam aspect ratios $(L / D)$, which varies from 1 to 4 .

\section{Boundary conditions}

The two types of loading considered are: parabolic shear loading with maximum intensity of $\sigma_{0}$ at the right lateral edge and uniformly distributed normal loading with intensity $\sigma_{0}$ at the top surface. With different combinations of supporting and loading conditions several cases are considered here. 


\section{Results and discussion}

\section{General}

The effect of fiber orientation on the state of stresses, specially at the supporting end of a fiberreinforced composite cantilever beam is investigated for two limiting cases of fiber orientations, i.e., $\theta=0^{\circ}$ and 90 . In order to make the investigation comprehensive, the influence of a number of practical issues, such as supporting condition, loading condition, fiber stiffness etc. is taken into consideration. For most of the cases, the fiber orientation effect is demonstrated as a function of beam aspect ratio $(L / D)$, which is varied over a range of 1 4. The stresses are normalized with respect to the maximum intensity of applied loading $\left(\sigma_{0}\right)$ and plotted against the normalized position along the beam depth. For the sake of present analysis, the maximum change in stress field caused by the change of fiber orientation from $\theta=0^{\circ}$ and 90 is considered as the indicator of the effect of fiber orientation. The finite difference meshnetwork used to model the cantilever beam with different aspect ratios was constructed by taking [(41 55) $\times 25]$ nodal points along $x$ and $y$-axis, respectively. For all the cases of loadings, the maximum intensity of the loading is taken as $3 \mathrm{MPa}$. For most of the results, glass/epoxy composite with fiber volume fraction $V_{f}=0.45$ is considered as the beam material, unless its mentioned otherwise. The various mechanical properties of glass/epoxy as well as graphite/epoxy composite material with different fiber volume fractions are listed in Table 1.

\section{The cantilever with global support under a parabolic end shear (Case-I)}

Case-I refers to a cantilever beam with conventional global support subjected to parabolic shear loading at the free lateral end, as shown in Figure 2. For Case-I the top and bottom surfaces are free from any type of loading. So, the normal and tangential components of loading are zero at these surfaces. The right lateral end is subjected to only a parabolic shear loading and the normal loading to the boundary is zero. The left lateral end is rigidly fixed to simulate the 
condition of global support i.e., displacements along both the normal and tangential directions are zero.

Mathematically the boundary conditions can be stated as follows:

At top surface $[x / D=0,0 \leq y / L \leq 1]: \sigma_{x x}(x, y)=\sigma_{x y}(x, y)=0$

At bottom surface $[x / D=1,0 \leq y / L \leq 1]: \sigma_{x x}(x, y)=\sigma_{x y}(x, y)=0$

At right lateral end $[y / L=1,0 \leq x / D \leq 1]: \sigma_{y y}(x, y)=0 ; \sigma_{x y}(x, y)=4 \sigma_{0}\left[\frac{x}{D}-\left(\frac{x}{D}\right)^{2}\right]$

At left lateral end $[y / L=0,0 \leq x / D \leq 1]: u_{x}(x, y)=u_{y}(x, y)=0$

Figure 3 illustrates the lateral stress distribution along the beam depth at the supporting end of the beam $(y / L=0)$ as a function of beam aspect ratios ranging from 1 to 4 . It is seen that lateral stress assumes an anti-symmetric distribution. As the aspect ratio $(L / D)$ increases, the magnitude of stress increases for both the cases of $\theta=0^{\circ}$ and $90^{\circ}$, in which the beam with $\theta=$ $0^{\circ}$ always remains more critical in terms of lateral stress. It is also seen that the effect of fiber orientation, i.e., the difference between the maximum stresses corresponding to the two limiting cases of fiber orientation increases with the increase of aspect ratio. The effect is found to be most prominent around the corner points of the supporting end. For sections away from the supporting ends, such as $y / L=0.25$ and 5.0, the effect of fiber orientation is found to decrease with increase of $L / D$ ratio, which is in contrast with that at the supporting end of the beam. Not only that, at these sections lateral stress becomes higher for the case of $\theta=90^{\circ}$ than that with $\theta=0^{\circ}$.

It is however evident from the results that shear stress at the supporting end remains nearly unchanged for the case of $\theta=90^{\circ}$, while that with $\theta=0^{\circ}$ decreases with the increase of $L / D$ ratio. Figure 4 describes the distributions of shear stress over the supporting end as a function of beam aspect ratio. Similar to the case of lateral stresses, the difference between the 
magnitudes of shear stresses caused by fiber orientation is found to increase with the increase of beam aspect ratio. For sections away from the supporting end, the effect of fiber orientation decreases as $L / D$ increases.

The present investigation reveals that the distribution of shear stress at the supporting end of the composite cantilever differs significantly from the standard parabolic one. This parabolic distribution is only achieved at a section sufficiently away from the supporting end. This developing distance from the supporting end is identified to be dependent on fiber orientation. The composite cantilever with fiber orientation, $\theta=0^{\circ}$ assumes the standard parabolic profile of shear stress at an early stage compared to the case of $\theta=90^{\circ}$ fiber.

Figure 5 describes the distribution of bending stress at the supporting ends of short composite cantilevers as a function of beam aspect ratio. This bending stress is identified to be the most important one in terms of magnitude than the other two components. For both the fiber orientations bending stress increase with the increase of aspect ratio. At any lateral section, bending stress is higher for the case of $\theta=90^{\circ}$ than that with $\theta=0^{\circ}$. Most importantly, the difference in the stress level caused by the change in fiber orientation is found to decrease with the increase of $L / D$ ratio, which is in contrast with those associated with lateral and shear stresses. For beam of $L / D=4$, the fiber orientation effect on the bending stress at the supporting end is not very insignificant, rather the magnitude of the stress for $\theta=90^{\circ}$ is about $15 \%$ higher than that with $\theta=0^{\circ}$. Another important observation is that the effect of fiber orientation on bending stress is highly localized, that is, the effect is found prominent only around the two corner regions of the support (see Figure 5). Further, the distribution at the supporting end deviates from the standard linear distribution, however the deviation decreases with the increase of distance from the supporting end. Analysis of the results also reveals that the beam with fiber orientation $\theta=90^{\circ}$ causes the bending stress distribution at a particular transverse section to be more nonlinear than that with $\theta=0^{\circ}$. The effect of fiber orientation on the overall 
state of stress in a short composite cantilever is significant primarily around the supporting end and, for sections sufficiently away from the end, the effect is almost insignificant.

\section{The cantilever under parabolic end shear with a distributed local support}

\section{(Case-II)}

In this section, fiber orientation effect is investigated for a special type of support applied to the same cantilever, namely distributed local support, the schematic of which is illustrated in Figure 6, and is referred from here as case-II. The boundary conditions used to solve the cantilever (Case-II) are almost the same as that of Case-I. except those on the left supporting end. Instead of the conventional global fixed support, distributed local supports of equal spacing are adopted at the left lateral end. For the fixed portions of the support, the boundary conditions remain the same as that of global support, i.e., $u_{x}=0$ and $u_{y}=0$, and for the free segments the corresponding conditions are $\sigma_{y y}=0$ and $\sigma_{x y}=0$. The extreme nodal points of the fixed portion i.e. the nodal points in between a fixed and free portion are taken as fixed points and condition for fixed point i.e. displacement boundary condition is applied at these nodal points.

Figure 7 illustrates the lateral stress distributions at the supporting end of the beam as a function of beam aspect ratio. Although the overall anti-symmetry of the lateral stress distributions are verified, the distribution pattern differs significantly from that of the global support. Sudden jumps of stresses are observed around the extreme points of fixed portions of the support, which is mainly due to the singularity effect. In The overall magnitude of the lateral stress for both the cases of fiber orientation as well as the corresponding difference between the stresses is found to be higher when compared with the corresponding results of global support described in Figure 3. For the case of fiber orientation, $\theta=90^{\circ}$, no significant change in the distributions of lateral stress at the supporting end is observed with the increase of $L / D$ ratio, however, the 
stress level increases quite significantly for the case of $\theta=0^{\circ}$. However, for the beam with global support, slight increase in magnitude of the lateral stress was observed when the aspect ratio was increased.

When the results at sections away from the supporting end are analyzed, it is observed that, the distribution of lateral stress for the cantilever with $\theta=90^{\circ}$ contains more information of the support than with $\theta=0^{\circ}$ fibers. In other word, the lateral stress distributions for the case of fiber orientation $\theta=0^{\circ}$ are less affected by the type of support adopted for the beam. At midspan section of the beams $(y / L=0.5)$, the state of lateral stresses is found to be very similar for both the cantilevers of Case-I and Case-II, where neither the fiber-orientation effect nor the influence of the type of support is significant on the lateral stress.

Figure 8 shows the distributions of shear stress at the supporting end of the cantilever (CaseII) as a function of fiber-orientation and beam aspect ratio. The shear-free conditions are accurately reproduced by the present solution for the free segments of the distributed support. Shear stresses are developed for the fixed segments of the support, which are however identified to be dependent on fiber orientation and $L / D$ ratio. Sharp rises of shear stress especially at the corners of the fixed portions are encountered in the distribution which are mainly due to the effect of singularity. Similar to the case of global support, the difference in shear stress caused by the change of fiber orientation is also found to increase with the increase of beam aspect ratio. Analysis of the results show that the cantilever with $\theta=0^{\circ}$ becomes more critical in terms of shear stress than that with $\theta=90^{\circ}$ fiber.

The anti-symmetric distribution of bending stress at the supporting end of the cantilever is shown in Figure 9, which reveals that the stress level increases with the increase of beam aspect ratio. The distributions of bending stress at the discretely supporting end are found to be less affected by the singularities associated with the terminal points of the support. Bending stresses 
are developed only at the fixed portions of the support, whereas the free portions are completely free from bending stresses. Most importantly, the effect of fiber orientation on the state of bending stress, especially at the supporting end of the cantilever (Case-II) is verified to be less significant when compared to the case of global support (Case-I), which can be visualized from the comparison of Figures 5 and 9. Similar to the cases of lateral and shear stresses, the effect of fiber orientation on the distribution of bending stress decreases as we move from the supporting end, and around the mid-span position, the effect is found to be almost insignificant for all the beams considered.

A review of the results of lateral and shear stress distributions as shown in Figures 7 and 8 shows that, at the free portions of the distributed local support, effect of fiber orientation is almost negligible. However, at the fixed or restrained portions of the supporting end, the effect is prominent. Based on the above observation, it can be expected that for a structural component like beam or plate, the effect of fiber orientation on stresses would be completely different if the associated boundaries are free from restraints. In order to investigate the possibility, a both

ends free beam of identical material was analyzed under pure bending for both the cases of fiber orientation. The investigation shows that fiber orientation has, in fact, no significant influence on any of the components of stress at any sections of the beam, which, in turn, reveals that the built-in supporting end of a cantilever beam is probably the primary reason to make the fiber orientation effect prominent on the elastic field.

\section{Effect of type of loading}

In this section it is checked how fiber orientation effect is influenced by the type of loading. For this a cantilever beam of the same material (glass/epoxy with $V_{f}=0.45$ ) with global support which is subjected to uniformly distributed normal loading of intensity $\sigma_{0}$ at the top surface is considered (Figure 10) which is stated as case-III. Figure 11 shows the lateral stress distribution 
along the beam depth at the supporting end. It is seen that as aspect ratio increases, effect of fiber orientation increases as seen in case-I. But for any particular aspect ratio, comparing Figure 3 and Figure 11 it is seen that in the case of normal loading both the magnitude and the effect of fiber orientation is much higher compared to that of parabolic shear loading. It is obvious that due to different type of loading condition the magnitude of stress will be different, but it is unexpected that the effect of fiber orientation will be different. Similarly comparing Figures 4 and 12 for shear stress and Figures 5 and 13 for bending stress, it can be concluded that at the supporting end higher effect of fiber orientation on all components of stress is seen for uniformly distributed normal loading than that of parabolic shear loading. Bending stress is highly influenced by the type of loading. Reason for higher effect of fiber orientation for this type of loading is that in this case load is acting directly on the fibers. Investigation is done at other lateral sections away from the supporting end and it is revealed that effect of fiber orientation on all components of stress is independent of loading type.

\section{Effect of fiber stiffness}

The effect of fiber orientation on the critical stresses in a composite cantilever is evaluated here for fibers having different stiffness. In order to carry out the investigation, results of a particular cantilever beam are compared for fiber materials having different stiffness, embedded in the same matrix material with the same fiber volume fraction. The cantilever beam with conventional global support under uniform transverse loading (Case-III) is analyzed here for two different composite materials, namely glass/epoxy and graphite/epoxy with identical value of fiber volume fraction, $V_{f}=0.68$. The corresponding mechanical properties of the composites are also included in the Table 1.

Figures 14 to 18 show the results obtained for the cantilever made of glass/epoxy and graphite/epoxy composites in a comparative fashion. Figure 14 shows the distributions of 
lateral stress at the supporting end for both materials with beam aspect ratios of 2 and 4 . When the results of individual materials are analyzed, it is observed that the stress level for both the materials as well as fiber orientations is influenced significantly by the size of the beam, as the magnitude is found to increase for all the cases with the increase of beam aspect ratio. From the comparison of results of Figures 14(a1) and (b1) or 14(a2) and (b2) it is realized how the fiber orientation effect is influenced by the stiffness of fiber.

When the results of glass/epoxy and graphite/epoxy beams are compared for a particular aspect ratio, it is observed that the distribution of lateral stress at the supporting end remains nearly unchanged if the fibers are oriented along the lateral direction of the beam ( $x$-axis). However, the corresponding stress level is found to change quite significantly when the fibers are situated along the axis of the beam ( $y$-axis). More specifically, the magnitude of the corresponding maximum lateral stress in a cantilever is found to decrease with the increase of fiber stiffness. Therefore, the overall change in magnitude of the stress caused by the change of fiber orientation increases with the increase of fiber stiffness in the same matrix material.

Similar to the case of lateral stress, shear stress level at the supporting end of the graphite/epoxy cantilever is found to be higher than that in the glass/epoxy cantilever as illustrated in Figure 15. However, this increase in stress level is identified to be a function of fiber orientation. More specifically the increase in stress due to the increase in fiber stiffness is relatively higher for the case of $\theta=90^{\circ}$ than that with $\theta=0^{\circ}$. Therefore, the overall change in the maximum shear stress at the supporting end of the cantilever caused by the change in fiber orientation from $\theta$ $=0^{\circ}$ to $\theta=90^{\circ}$ is found to increase, to some extent, with the increase in fiber stiffness, which is however influenced quite substantially by the beam aspect ratio [see Figures 15(b1) and (b2)]. 
Figure 16 describes the distribution of bending stress at the supporting end of the cantilever for the two fiber-reinforced composite materials as a function of beam aspect ratio. It is observed from the results that, for a particular composite material, the change in stress level caused by the change in fiber orientation is not much affected by the beam aspect ratio, even if associated fiber is of high stiffness. But for a particular aspect ratio of the cantilever, the effect of fiber orientation on the bending stresses at the supporting end is found to be influenced significantly by the fiber stiffness. A quantitative comparison of the results of Figures 16(a1) and (a2) reveals that the difference in bending stresses caused by the change of fiber orientation is nearly ten times higher when glass/epoxy is replaced by graphite/epoxy for the cantilever beam, $L / D=4$.

Now, some of the results of fiber orientation effect for sections away from the supporting end of a particular cantilever are presented in Figures 17 and 18. Figure 17 describes the distribution of shear stresses at sections $y / L=0.25$ and 5.0 of the cantilever (Case-III, $L / D=2$ ) for both the glass/epoxy and graphite/epoxy composites. Comparison of results of the two composites reveals that, for both the lateral sections, fiber orientation effect is more prominent for the case of graphite/epoxy than that with glass/epoxy. It can be stated in other words that the fiber orientation effect is influenced by the fiber stiffness for sections sufficiently away from the supporting end, as the two materials here differ mainly in terms of the stiffness of fibers (see Table 1). For the graphite/epoxy cantilever, the effect of fiber orientation is found to decrease as we move away from the supporting end, however, considerable effect is found to be remained even at the mid-span section of the beam, which is in contrast with the glass/epoxy beam. It can be mentioned here that the effect on shear stress is not fully insignificant at the mid-span section of a graphite/epoxy beam with $L / D=4$.

The corresponding results of bending stress for sections away from the supporting ends of glass/epoxy and graphite/epoxy cantilevers are shown in Figure 18. The effect of fiber orientation for both the lateral sections of the glass/epoxy beam is almost absent as mentioned 
earlier. However, for the graphite/epoxy beam, the scenario is quite different. In this case, the bending stress distributions, even around the mid-span section of the beam $(L / D=2)$ are highly nonlinear for the fiber orientation $\theta=90^{\circ}$; however, the corresponding distribution for the case of $\theta=0^{\circ}$ is found to resemble the standard linear characteristic even at $y / L=0.25$ section. The nonlinear characteristic of bending stress distribution for graphite/epoxy cantilever is also found to exist even for a beam with $L / D=4$. It is thus verified from the present analysis that fiber stiffness plays an important role in defining the effect of fiber orientation on the overall state of stress in short composite beams.

\section{Verification of accuracy}

Attempt is made here to compare some of the present solutions with those of usual computational technique. Exact analytical solution to the present problem of composite cantilever is hardly available in the literature, and that is why soundness as well as accuracy of the present displacement-potential solutions is verified by comparing them with those of finite element method, a widely used computational technique for structural analysis, which is however based on a philosophy completely different from the present one. For the purpose of comparison case-III has been chosen. Therefore, the problem of composite cantilever beam with global support subjected to uniformly distributed lateral loading has been solved by FEM using the standard commercial software ANSYS with an aspect ratio of $L / D=2$. Since the geometry of the beam is of rectangular shape, the four nodded rectangular plane elements (orthotropic) are used to construct the corresponding FEM mesh-network. The total number of finite elements used to discretize the beam was $10000(100 \times 100)$.

Figure 19 presents the comparison of stress distributions at the supporting end of a glass/epoxy cantilever beam $(L / D=2)$ for both fiber orientations of $\theta=0^{\circ}$ and $\theta=90^{\circ}$. Solutions for both FDM and FEM are presented in the same graph for the convenience of comparison. The 
supporting end is chosen as the section for comparison since the fixed end of a short cantilever is identified to be the most critical section in terms of stresses. For all the stress components, the two solutions are found to be in good agreement with each other, showing some discrepancy mainly near the two corner regions of the fixed support. From Figure 19 (a) it is seen that FDM solutions of lateral stress shows a normalized value of -1 at the top and 0 at the bottom surface, which appropriately reflects the associated given physical condition (see Table 2). Again, from Figure 19 (b), it is observed that shear stress has a value of zero at both top and bottom surfaces which is also in complete conformation to the actual boundary conditions. The discrepancy between the results of FEM and FDM is mainly due to the fact that the corner modeling schemes of these two methods are different. In case of the present FDM solutions, a total of three conditions out of the available four are satisfied appropriately at each of the corner points, which is however not the case for FEM solutions. ${ }^{19}$ FEM considers the corner points as the extreme points of the fixed support only, and accordingly the restrained boundary conditions are only satisfied. The distributions of bending stresses at the supporting end of the composite cantilever are compared in Fig. 19(c). The comparison of the results show that the two solutions (FDM and FEM) for both the cases of fiber orientation are in excellent agreement with each other both in terms of magnitude and nature of variation.

In the present finite-difference computational scheme, each corner points are considered as the common point to the connecting two boundaries. The potential of the present computational scheme is again extended to solve the same composite cantilever considering the corner points of the supporting end (A and B in Figure 10) as the terminal points of the fixed support only and free from any external loading, as modeled in the usual FEM solution. It would be worth mentioning here that the present computer program has been developed in such a flexible fashion that it can handle any combination/type of boundary conditions, especially for the points of singularity, which is, however, in general, out of the scope of available commercial 
FEM software. The two FDM solutions of lateral stress along with that of FEM are shown in Figure 19(a). The alternate FDM solution of the lateral stress is found to be in very good conformity with the corresponding FEM solution. It is important to mention here that, although the alternate FD modeling causes significant change in the distribution of lateral stresses especially at the corner regions of the fixed support, the corresponding distributions of shear and bending stresses are found to remain unchanged from their original FDM solutions.

The FD predictions for sections away from the supporting end are also compared with the corresponding FE solutions. The comparison shows that the solutions of all stress as well as displacement components obtained by the two computational methods, are almost identical in shape and magnitude, although relatively course meshes are used in the present FD discretization. The comparative analysis thus verifies the present displacement potential computational solutions of short composite cantilevers to be highly accurate and are founded on sound philosophy.

\section{Conclusions}

The effect of fiber orientation on the state of stresses in a short composite cantilever beam has been investigated in the perspective of beam aspect ratio, type of loading and supporting conditions as well as fiber stiffness. The solution is obtained using the displacement potential computational method. Both the qualitative and quantitative results of different stress components, obtained for two limiting cases of fiber orientation, at different sections of the beam provide explicit information of the effect of fiber orientation, especially on the critical stresses in the fiber-reinforced composite cantilever beam.

Salient features of the investigation are summarized as follows: 
- The supporting end of a short composite cantilever beam is identified to be the most critical section in terms of stresses.

- The effect of fiber orientation is verified to be influenced quite significantly by the beam aspect ratio, type of loading and supporting conditions as well as fiber stiffness.

- The effect of fiber orientation is found to be prominent primarily at the supporting end of the beam and, for most cases, it decreases drastically with the increase of distance from the supporting end.

- The effect of fiber orientation on lateral and shear stresses becomes more prominent for higher aspect ratios of the beam, while an opposite characteristic is encountered for the case of bending stresses.

- The overall change in the state of stresses caused by the change in fiber orientation from $\theta=0^{\circ}$ to $90^{\circ}$ is found to enhance for fiber materials having higher stiffness. In addition, fiber stiffness is found to play a dominant role in defining the zone adjacent to the supporting end, over which the fiber orientation effect is prominent. 


\section{References}

1. Rehfield, L. W. \& Murthy, P. L. N. Toward a new engineering theory of bendingFundamentals. AIAA J. 20, 693-699 (1982).

2. Goodier, J. N. \& Timoshenko, S. Theory of elasticity. (McGraw-Hill, 1979).

3. Murty, A. V. K. Toward a consistent beam theory. AIAA J. 22, 811-816 (1984).

4. Chow, L., Conway, H. D. \& Winter, G. Stresses in deep beams. Trans. Am. Soc. Civ. Eng. 118, 686-702 (1953).

5. Dow, J. O., Jones, M. S. \& Harwood, S. A. A new approach to boundary modelling for finite difference applications in solid mechanics. Int. J. Numer. Methods Eng. 30, 99113 (1990).

6. Hardy, S. J. \& Pipelzadeh, M. K. Static analysis of short beams. J. strain Anal. Eng. Des. 26, 15-29 (1991).

7. Ahmed, S. R., Idris, A. B. M. \& Uddin, M. W. Numerical solution of both ends fixed deep beams. Comput. Struct. 61, 21-29 (1996).

8. Ahmed, S. R., Khan, M. R., Islam, K. M. S. \& Uddin, M. W. Investigation of stresses at the fixed end of deep cantilever beams. Comput. Struct. 69, 329-338 (1998).

9. Bernasconi, A., Davoli, P., Basile, A. \& Filippi, A. Effect of fibre orientation on the fatigue behaviour of a short glass fibre reinforced polyamide-6. Int. J. Fatigue 29, 199-208 (2007).

10. Nath, S. K. D. \& Afsar, A. M. Analysis of the effect of fiber orientation on the elastic field in a stiffened orthotropic panel under uniform tension using displacement 
potential approach. Mech. Adv. Mater. Struct. 16, 300-307 (2009).

11. Naghipour, P., Bartsch, M., Chernova, L., Hausmann, J. \& Voggenreiter, H. Effect of fiber angle orientation and stacking sequence on mixed mode fracture toughness of carbon fiber reinforced plastics: Numerical and experimental investigations. Mater. Sci. Eng. A 527, 509-517 (2010).

12. Kaman, M. O. Effect of fiber orientation on fracture toughness of laminated composite plates [0/0] s. Eng. Fract. Mech. 78, 2521-2534 (2011).

13. Majid, D. L., Abdullah, E. J., Harun, N. F., Lim, G. Y. \& Baharudin, B. Effect of fiber orientation on the structural response of a smart composite structure. Procedia Eng. 50, 445-452 (2012).

14. Vincent, T. \& Ozbakkaloglu, T. Influence of fiber orientation and specimen end condition on axial compressive behavior of FRP-confined concrete. Constr. Build. Mater. 47, 814-826 (2013).

15. Akanda, M. A. S., Ahmed, S. R. \& Uddin, M. W. Stress analysis of gear teeth using displacement potential function and finite differences. Int. J. Numer. Methods Eng. 53, 1629-1640 (2002).

16. Reaz Ahmed, S., Deb Nath, S. K. \& Wahhaj Uddin, M. Optimum shapes of tire-treads for avoiding lateral slippage between tires and roads. Int. J. Numer. Methods Eng. 64, 729-750 (2005).

17. Ahmed, S. R., Hossain, M. Z. \& Uddin, M. W. A general mathematical formulation for finite-difference solution of mixed-boundary-value problems of anisotropic materials. Comput. Struct. 83, 35-51 (2005). 
18. Deb Nath, S. K., Afsar, A. M. \& Reaz Ahmed, S. Displacement potential solution of a deep stiffened cantilever beam of orthotropic composite material. J. Strain Anal. Eng. Des. 42, 529-540 (2007).

19. Deb Nath, S. \& Ahmed, S. R. A displacement potential-based numerical solution for orthotropic composite panels under end moment and shear loading. J. Mech. Mater. Struct. 4, 987-1004 (2009).

20. Deb Nath, S. K. \& Wong, C. H. Finite-difference solution of a both-end-fixed orthotropic composite beam under uniformly distributed loading using displacement potential function formulation. J. Eng. Mech. 137, 258-267 (2011).

21. Nath, S. K. D., Ahmed, S. R. \& Afsar, A. M. Displacement potential solution of short stiffened flat composite bars under axial loading. Appl. Mech. Eng. 11, 557 (2006).

22. Modak, P., Hossain, M. J., Ikram, I. \& Ahmed, S. R. A comparative analysis of elastostatic responses of a cross-ply and angle-ply laminated panel using a singlevariable lamination theory. Procedia Eng. 90, 213-218 (2014).

23. Ahmed, S. R. \& Modak, P. Stress analysis of symmetric and anti-symmetric discretely stiffened laminated cantilever beams using displacement-potential field. Appl. Math. Comput. 258, 465-482 (2015).

24. Modak, P., Hossain, M. J. \& Ahmed, S. R. Analysis of a hybrid balanced laminate as a structural material for thick composite beams with axial stiffeners. AIP Conf. Proc. 1754, 030012 (2016).

25. Kaw, A. K. Mechanics of composite materials. (CRC press, 2005).

26. Jones, R. M. Mechanics of composite materials. (CRC press, 2018). 


\section{Nomenclature}

\begin{tabular}{|c|c|}
\hline$E_{f}$ & Modulus of elasticity of fiber material \\
\hline$E_{m}$ & Modulus of elasticity of matrix material \\
\hline$E_{1}$ & Longitudinal modulus of elasticity of composite \\
\hline$E_{2}$ & Transverse modulus of elasticity of composite \\
\hline$v_{f}$ & Poisson's ratio of fiber material \\
\hline$v_{m}$ & Poisson's ratio of matrix material \\
\hline$v_{12}$ & Major Poisson's ratio \\
\hline$v_{21}$ & Minor Poisson's ratio \\
\hline$G_{12}$ & In-plane shear modulus \\
\hline$V_{f}$ & Fiber volume fraction \\
\hline$u_{n}$ & Normal displacement component \\
\hline$u_{t}$ & Tangential displacement component \\
\hline$u_{x}$ & Displacement component in the $x$-direction \\
\hline$u_{y}$ & Displacement component in the $y$-direction \\
\hline$\sigma_{n}$ & Normal stress component \\
\hline$\sigma_{t}$ & Tangential stress component \\
\hline$\sigma_{x x}$ & Lateral stress or Normal stress component in the $x$-direction \\
\hline$\sigma_{y y}$ & Bending stress or Normal stress component in the $x$-direction \\
\hline$\sigma_{x x}$ & Shearing stress component in the $x-y$ plane \\
\hline$\theta$ & Fiber orientation i.e. the angle between fiber direction and $x$-direction \\
\hline$\psi$ & Displacement potential function \\
\hline$L, D$ & Length and width respectively \\
\hline
\end{tabular}




$$
\begin{aligned}
& \sigma_{0} \quad \text { Maximum intensity of parabolic shear load or intensity of uniformly } \\
& \text { distributed normal load applied per unit area }
\end{aligned}
$$

Table 1. Mechanical properties of composite materials used..$^{21,25}$

\begin{tabular}{ccccc}
\hline \multirow{2}{*}{ Material } & \multirow{2}{*}{ Property } & \multicolumn{3}{c}{ Composite } \\
\cline { 3 - 5 } & & $V_{f}=0.45$ & $V_{f}=0.68$ & Graphite/epoxy \\
\cline { 2 - 5 } Fiber & $E_{f}(\mathrm{GPa})$ & 85.52 & 85.52 & $V_{f}=0.68$ \\
\cline { 2 - 5 } & $v_{f}$ & 0.22 & 0.22 & 0.20 \\
\hline \multirow{2}{*}{ Matrix } & $E_{m}(\mathrm{GPa})$ & 3.45 & 3.45 & 3.45 \\
\cline { 2 - 5 } & $v_{m}$ & 0.35 & 0.35 & 0.35 \\
\cline { 2 - 5 } Composite & $E_{1}(\mathrm{GPa})$ & 38.6 & 59.31 & 648.32 \\
\cline { 2 - 5 } & $E_{2}(\mathrm{GPa})$ & 8.27 & 22.07 & 24.83 \\
\cline { 2 - 5 } & $G_{12}(\mathrm{GPa})$ & 4.14 & 8.97 & 0.25 \\
\cline { 2 - 5 } & $v_{12}$ & 0.26 & 0.26 & 0.010 \\
\cline { 2 - 5 } & $v_{21}$ & 0.056 & 0.097 & \\
\hline
\end{tabular}


Table 2. Boundary conditions used for corner points of the cantilever.

\begin{tabular}{|c|c|c|c|c|}
\hline \multirow{2}{*}{ Corner points } & \multirow{2}{*}{$\begin{array}{l}\text { Physical } \\
\text { parameters } \\
\text { available at the } \\
\text { corner points }\end{array}$} & \multirow{2}{*}{$\begin{array}{l}\text { Parameters } \\
\text { used to } \\
\text { model the } \\
\text { boundary } \\
\text { condition }\end{array}$} & \multicolumn{2}{|c|}{$\begin{array}{c}\text { Correspondence between mesh } \\
\text { points and given boundary } \\
\text { conditions }\end{array}$} \\
\hline & & & $\begin{array}{l}\text { Mesh point on } \\
\text { the physical } \\
\text { boundary }\end{array}$ & $\begin{array}{l}\text { Mesh point on } \\
\text { the imaginary } \\
\text { boundary }\end{array}$ \\
\hline $\begin{array}{c}\text { A } \\
(\text { Case }- \text { I and II })\end{array}$ & {$\left[\left(\sigma_{n}, \sigma_{t}\right),\left(u_{n}, u_{t}\right)\right]$} & {$\left[\sigma_{n}, \sigma_{t}, u_{n}\right]$} & $\sigma_{n}=0$ & $\sigma_{t}=0, u_{n}=0$ \\
\hline $\begin{array}{c}\mathrm{A} \\
(\text { Case - III) }\end{array}$ & {$\left[\left(\sigma_{n}, \sigma_{t}\right),\left(u_{n}, u_{t}\right)\right]$} & {$\left[\sigma_{n}, \sigma_{t}, u_{n}\right]$} & $\sigma_{n}=-\sigma_{0}$ & $\sigma_{t}=0, u_{n}=0$ \\
\hline $\mathrm{B}$ & {$\left[\left(\sigma_{n}, \sigma_{t}\right),\left(u_{n}, u_{t}\right)\right]$} & {$\left[\sigma_{n}, \sigma_{t}, u_{n}\right]$} & $\sigma_{n}=0$ & $\sigma_{t}=0, u_{n}=0$ \\
\hline $\begin{array}{c}\mathrm{C} \\
(\text { Case }-\mathrm{I} \text { and II })\end{array}$ & {$\left[\left(\sigma_{n}, \sigma_{t}\right),\left(\sigma_{t}, \sigma_{n}\right)\right]$} & {$\left[\sigma_{n}, \sigma_{t}, \sigma_{n}\right]$} & $\sigma_{n}=0$ & $\sigma_{t}=0, \sigma_{n}=0$ \\
\hline $\begin{array}{c}\mathrm{C} \\
\text { (Case - III) }\end{array}$ & {$\left[\left(\sigma_{n}, \sigma_{t}\right),\left(\sigma_{t}, \sigma_{n}\right)\right]$} & {$\left[\sigma_{n}, \sigma_{t}, \sigma_{n}\right]$} & $\sigma_{n}=0$ & $\sigma_{t}=0, \sigma_{n}=0$ \\
\hline $\begin{array}{c}\text { D } \\
(\text { Case }- \text { I and II })\end{array}$ & {$\left[\left(\sigma_{n}, \sigma_{t}\right),\left(\sigma_{t}, \sigma_{n}\right)\right]$} & {$\left[\sigma_{n}, \sigma_{t}, \sigma_{n}\right]$} & $\sigma_{n}=0$ & $\sigma_{t}=0, \sigma_{n}=0$ \\
\hline $\begin{array}{c}\mathrm{D} \\
(\text { Case - III) }\end{array}$ & {$\left[\left(\sigma_{n}, \sigma_{t}\right),\left(\sigma_{t}, \sigma_{n}\right)\right]$} & {$\left[\sigma_{n}, \sigma_{t}, \sigma_{n}\right]$} & $\sigma_{n}=-\sigma_{0}$ & $\sigma_{t}=0, \sigma_{n}=0$ \\
\hline
\end{tabular}

Case - I and II: Cantilever beam subjected to parabolic shear loading at right surface.

Case - III: Cantilever beam subjected to uniform normal loading at top surface. 


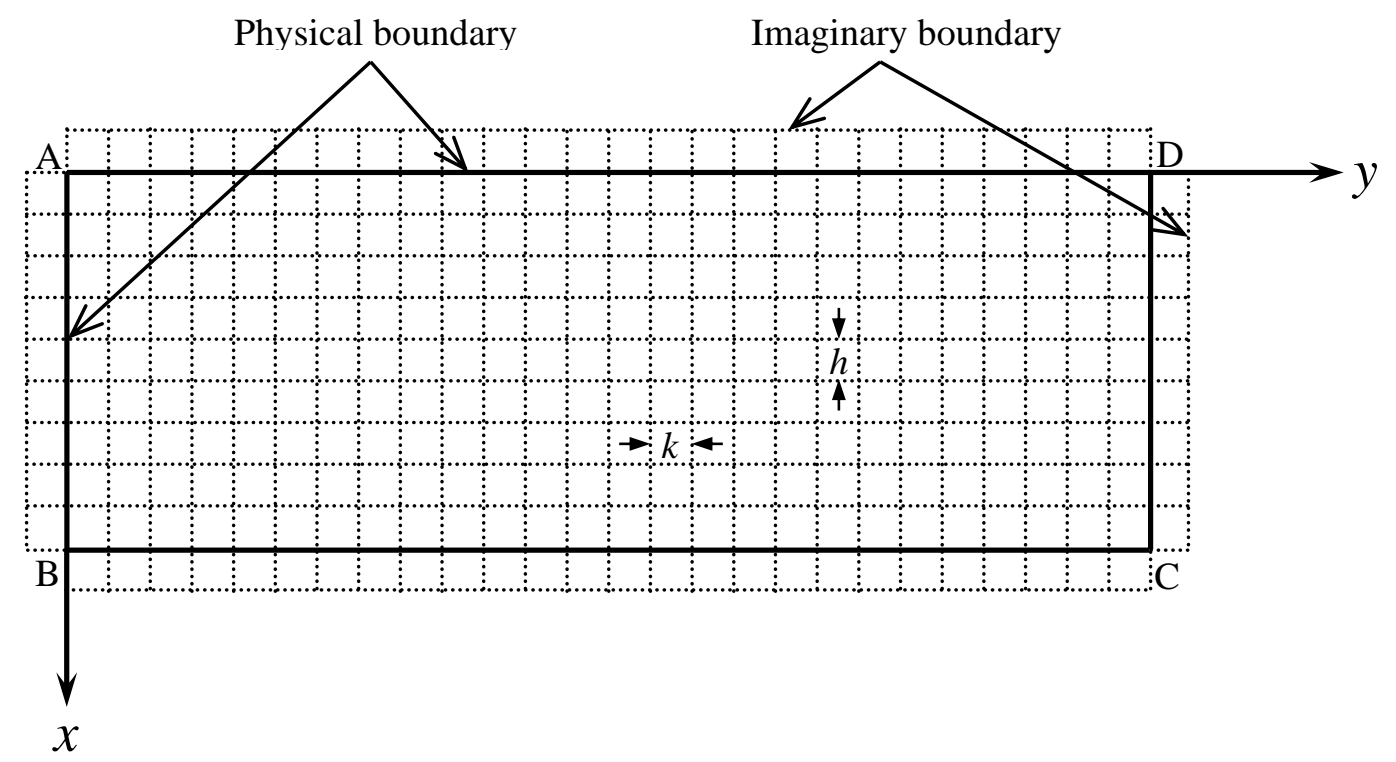

Figure 1. Finite difference discretization of the cantilever beam 


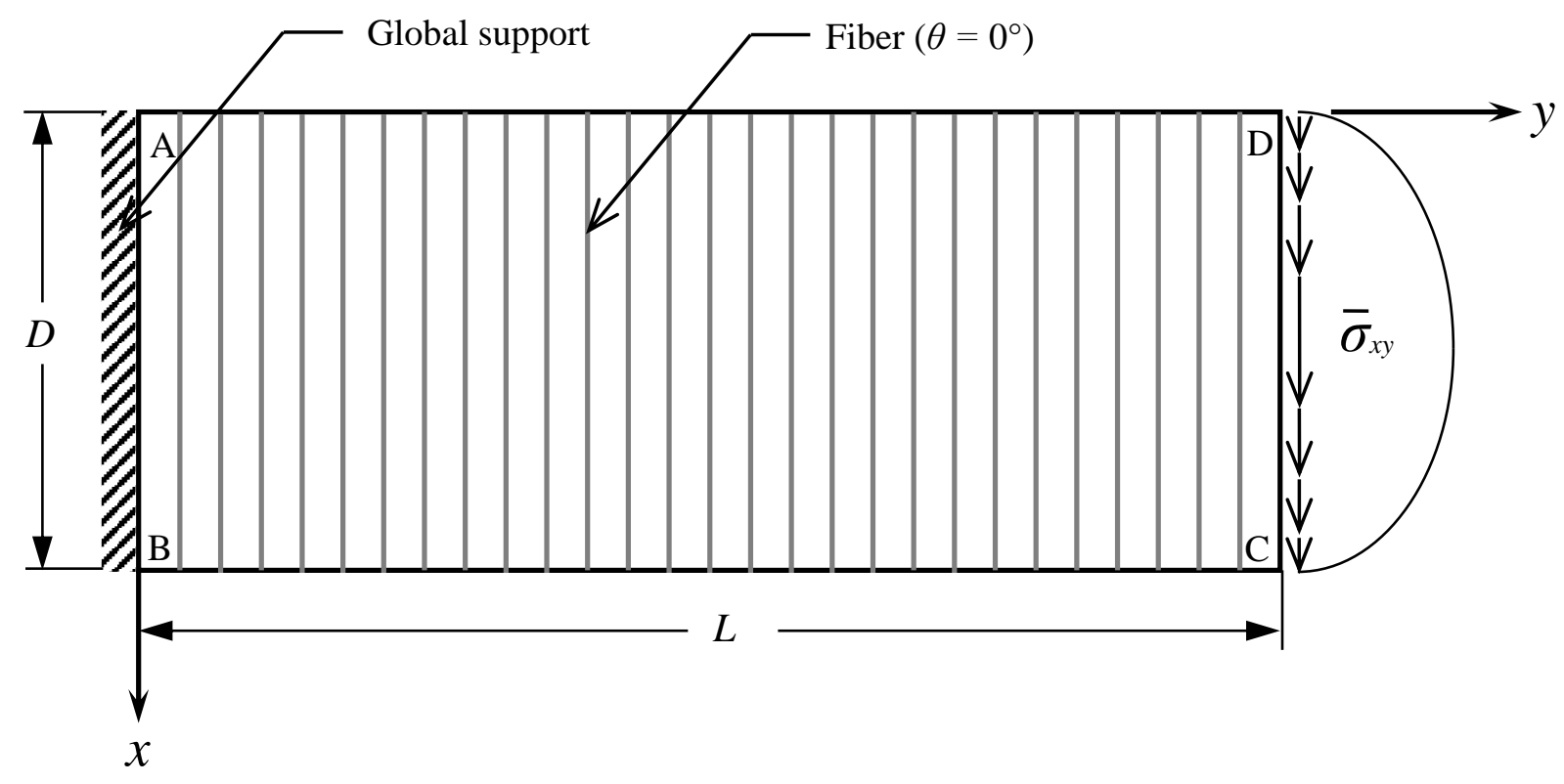

(a)

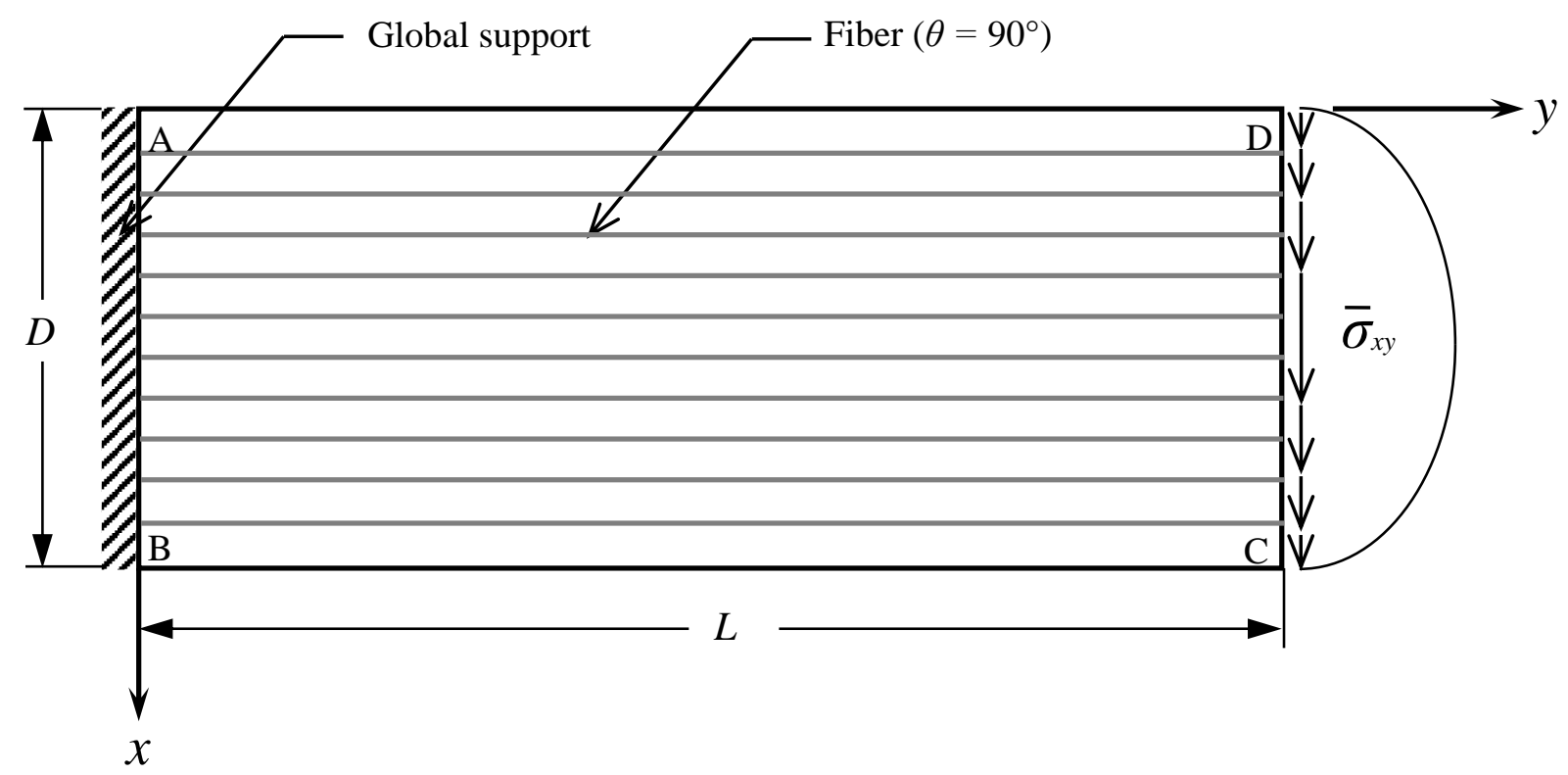

(b)

Figure 2. Cantilever beam with global support subjected to parabolic shear load with (a) $\theta=0^{\circ}$ and (b) $\theta=90^{\circ}$ 

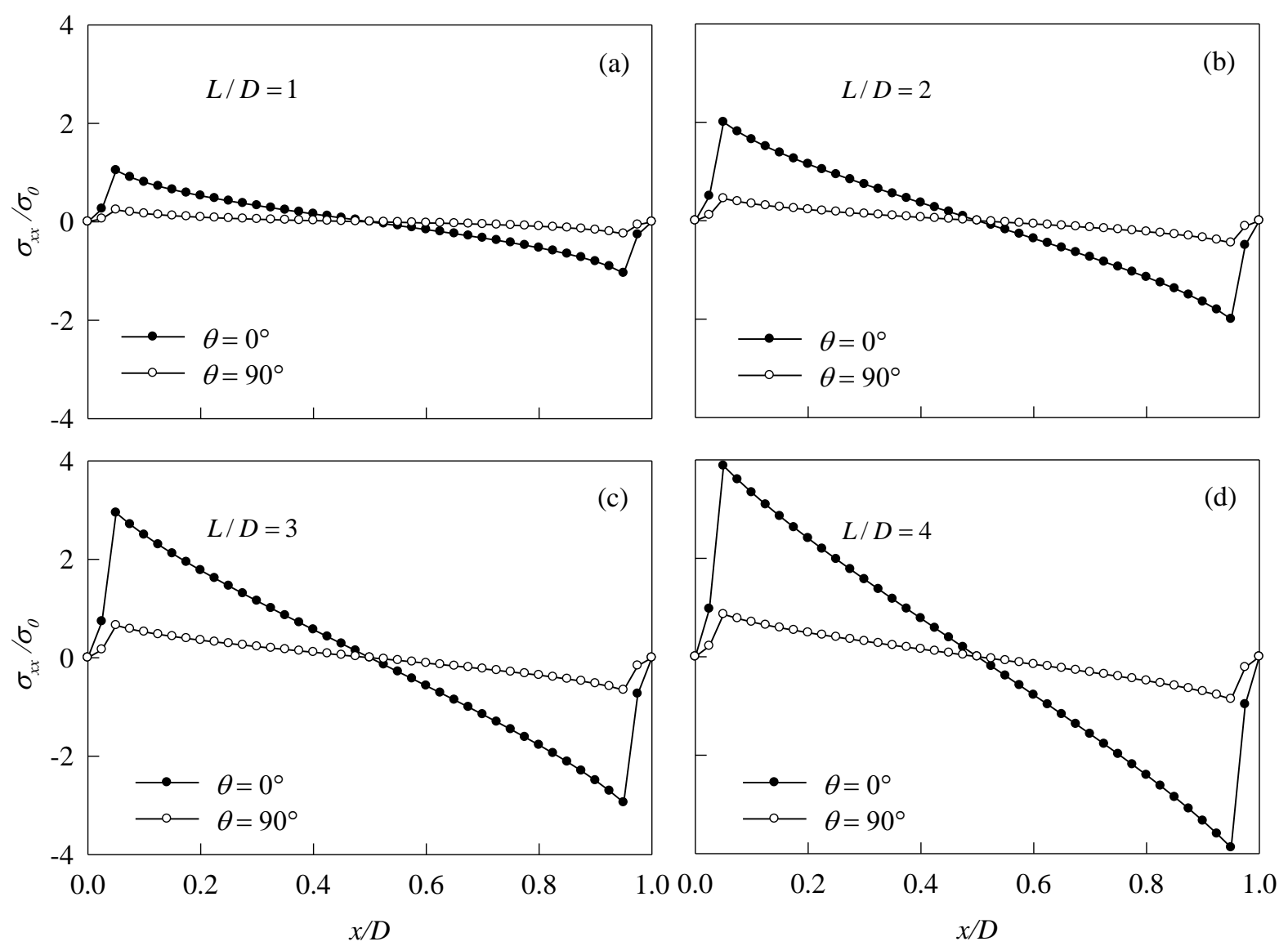

Figure 3. Distribution of lateral stress at the supporting end of the cantilever beam with global support under the parabolic end shear. 

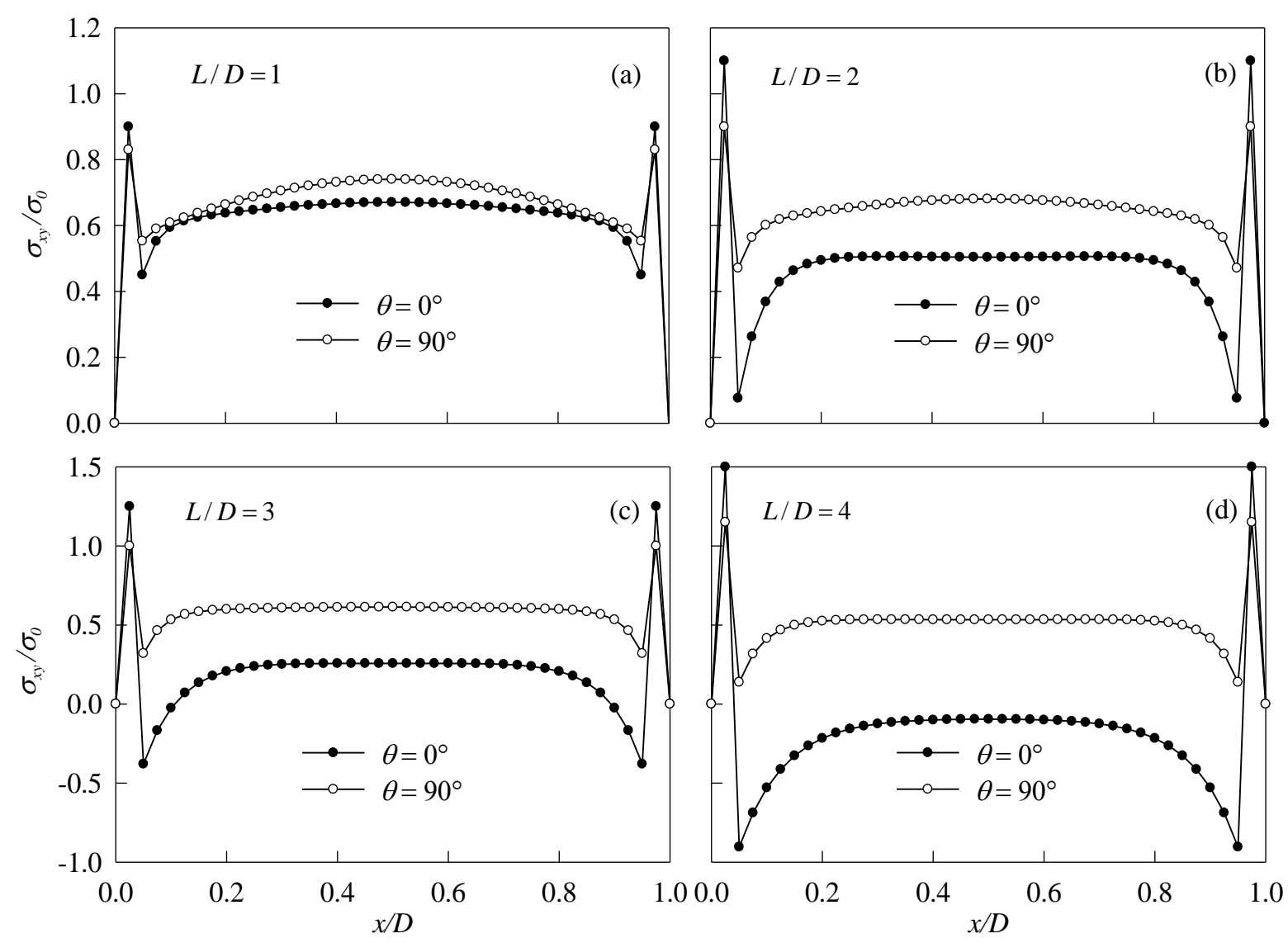

Figure 4. Distribution of shear stress at the supporting end of the cantilever beam with global support under the parabolic end shear. 

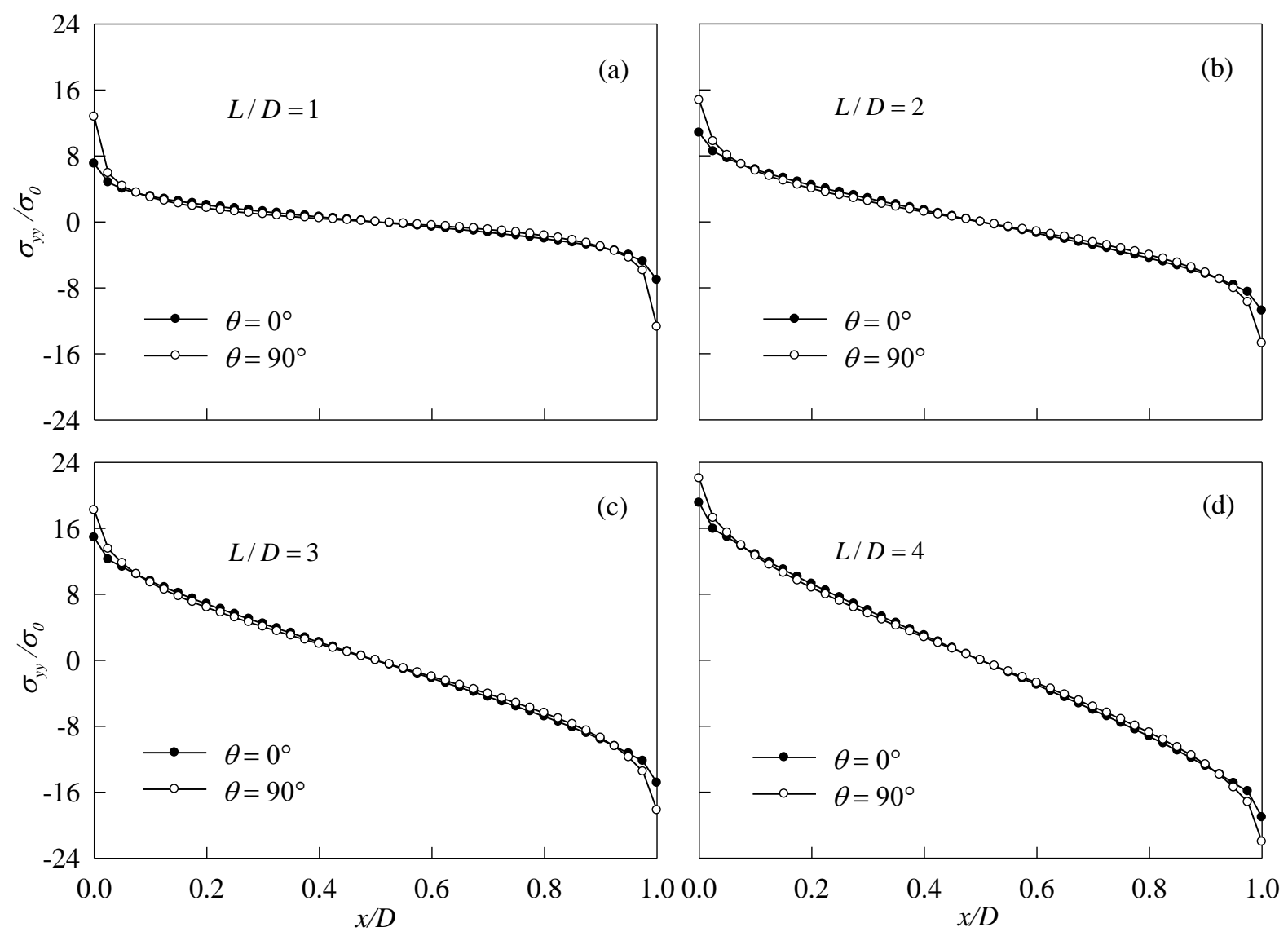

Figure 5. Distribution of bending stress at the supporting end of the cantilever beam with global support under the parabolic end shear. 


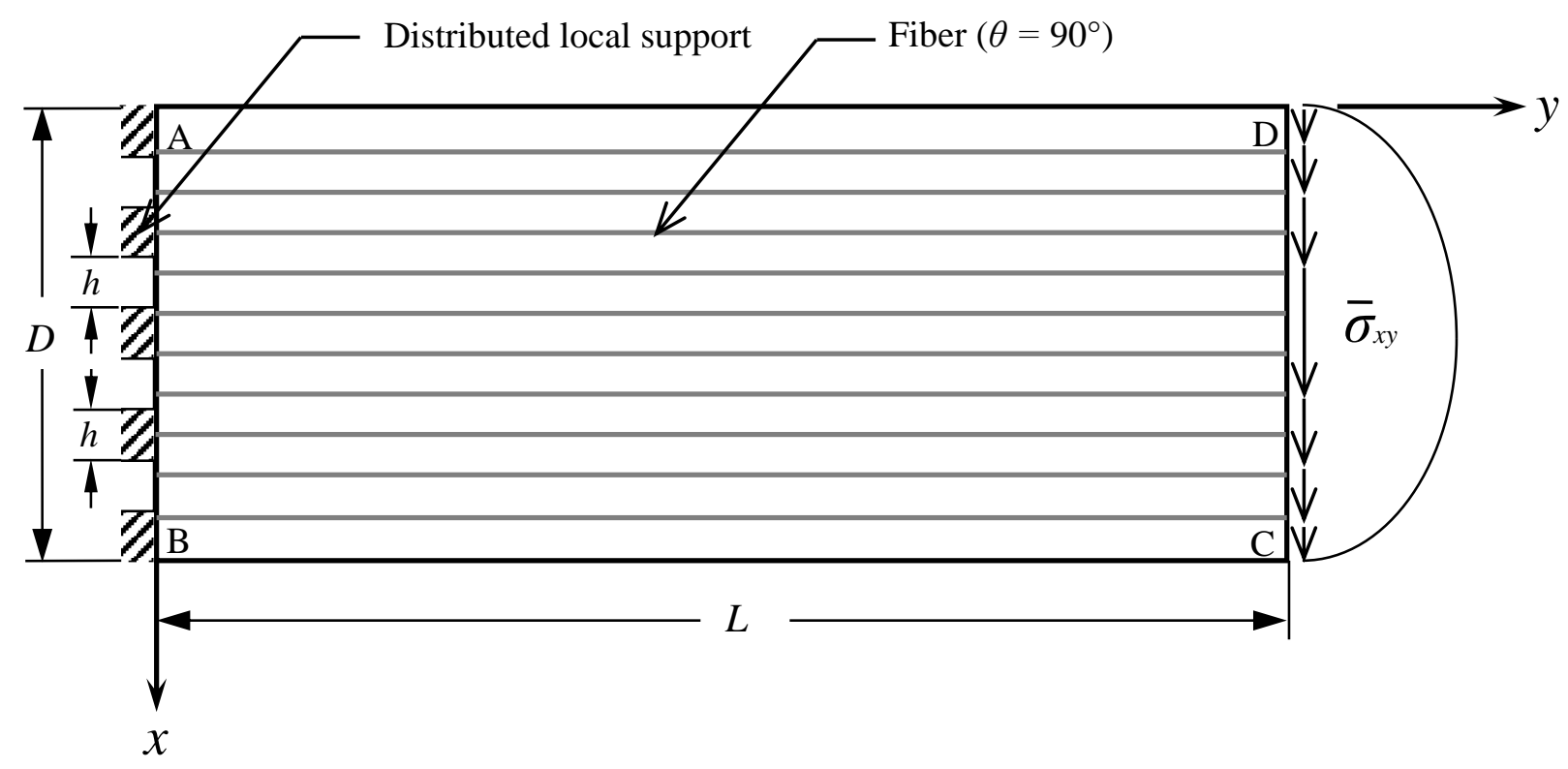

Figure 6. Cantilever beam with distributed local support subjected to parabolic shear load with $\theta=$ $90^{\circ}$ 

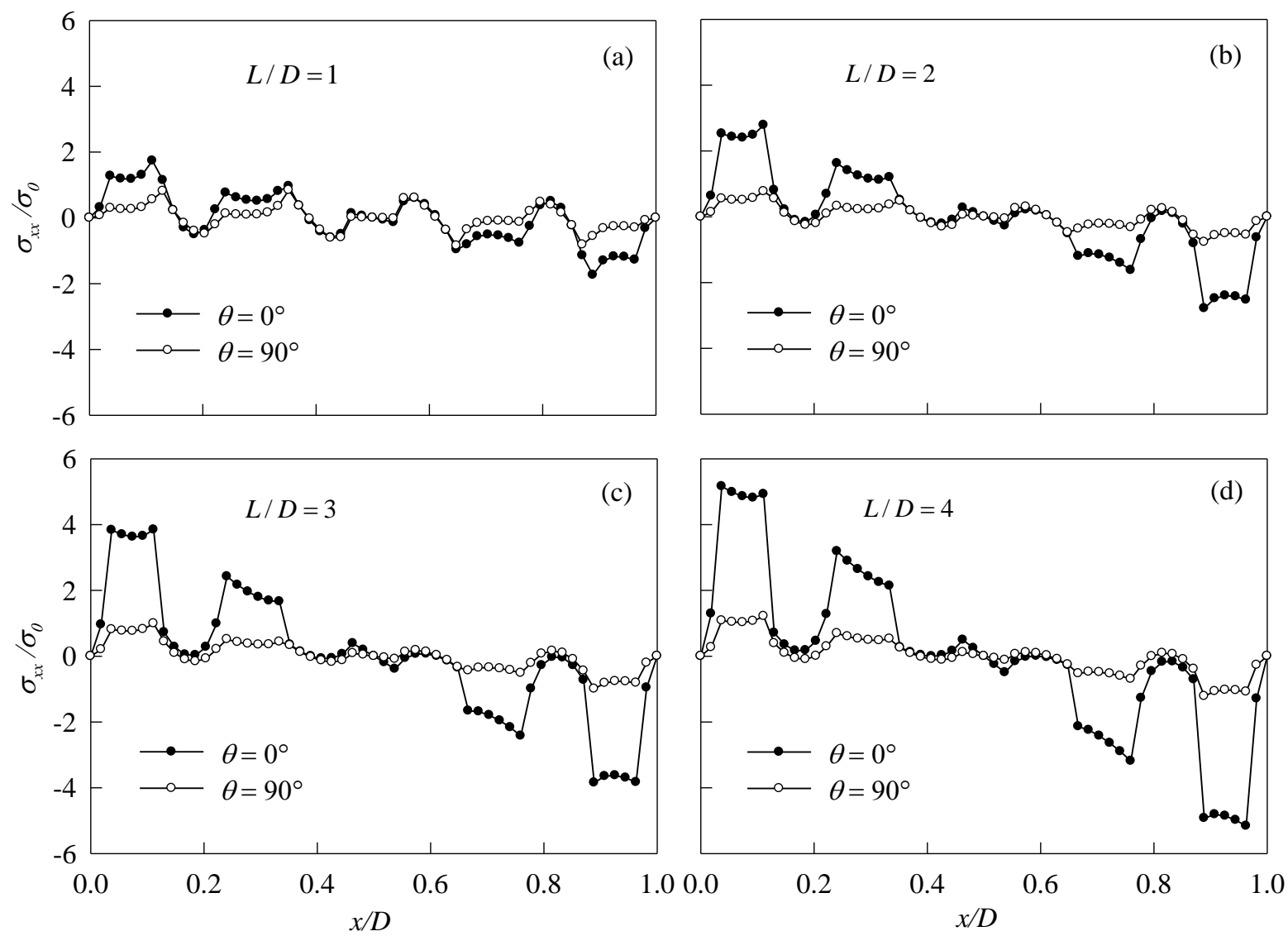

Figure 7. Distribution of lateral stress at the supporting end of the cantilever beam with distributed local support under the parabolic end shear. 

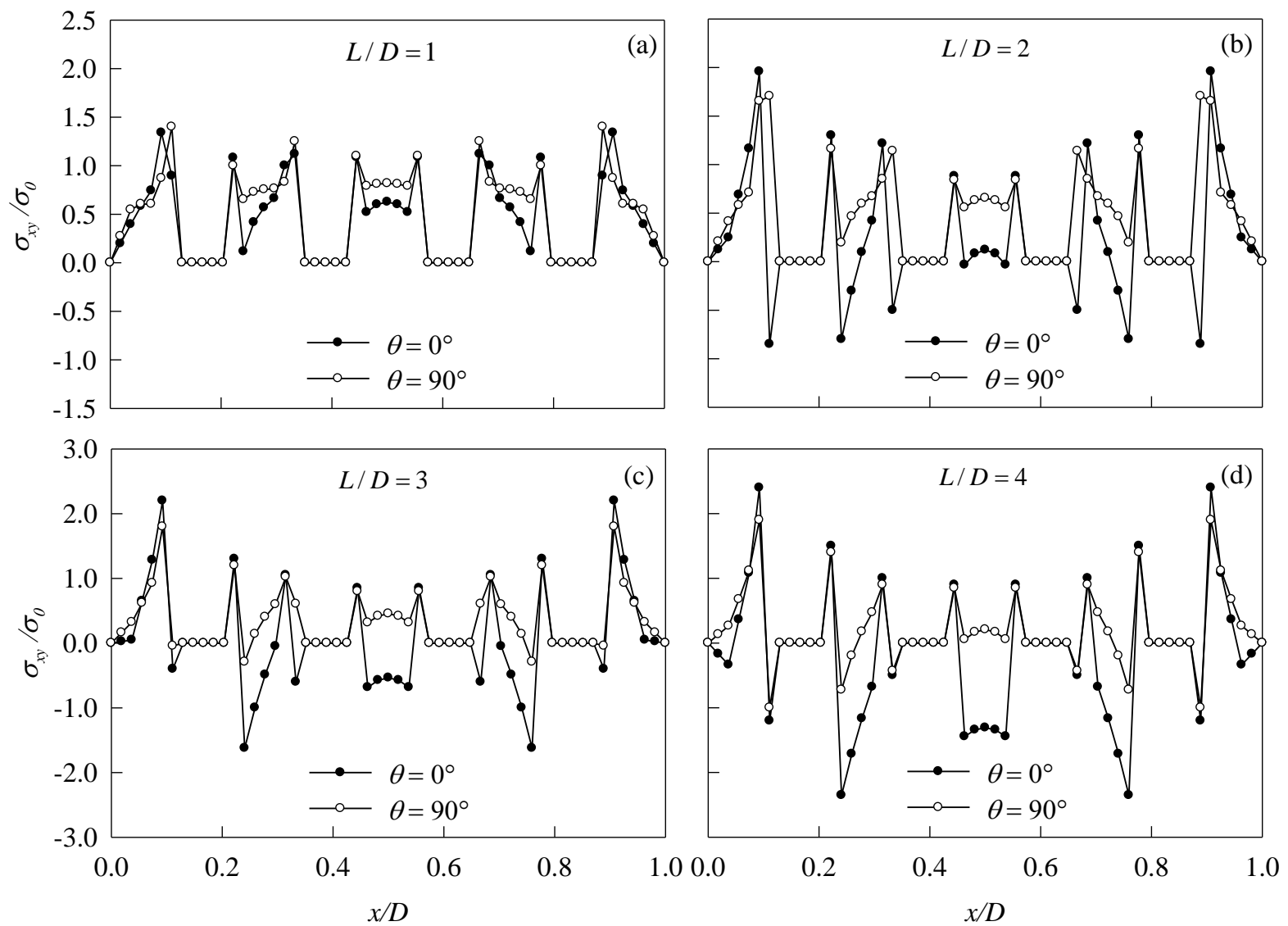

Figure 8. Distribution of shear stress at the supporting end of the cantilever beam with distributed local support under the parabolic end shear. 

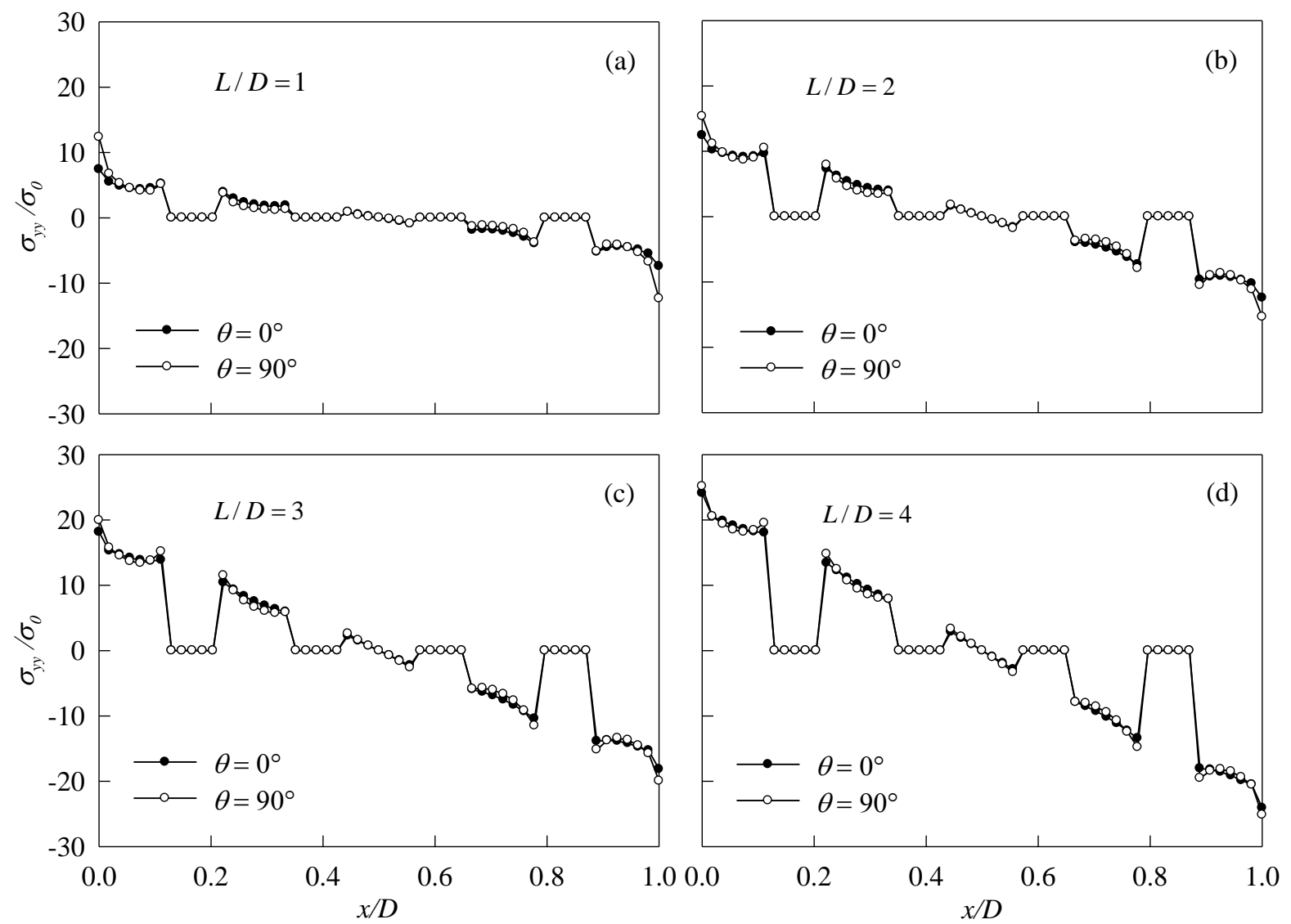

Figure 9. Distribution of bending stress at the supporting end of the cantilever beam with distributed local support under the parabolic end shear. 


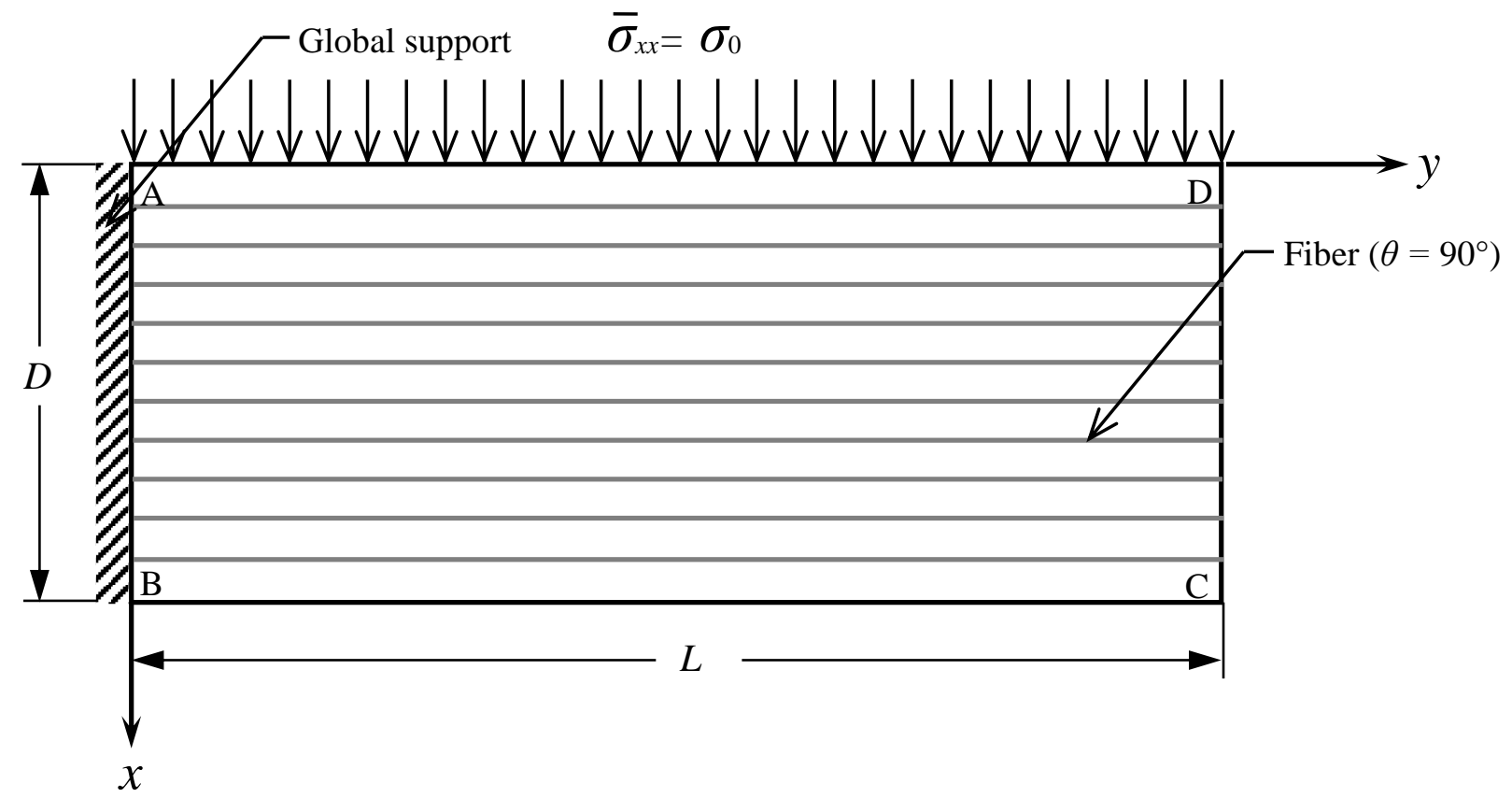

Figure 10. Cantilever beam with global support subjected to uniformly distributed loading with $\theta=$ $90^{\circ}$ 

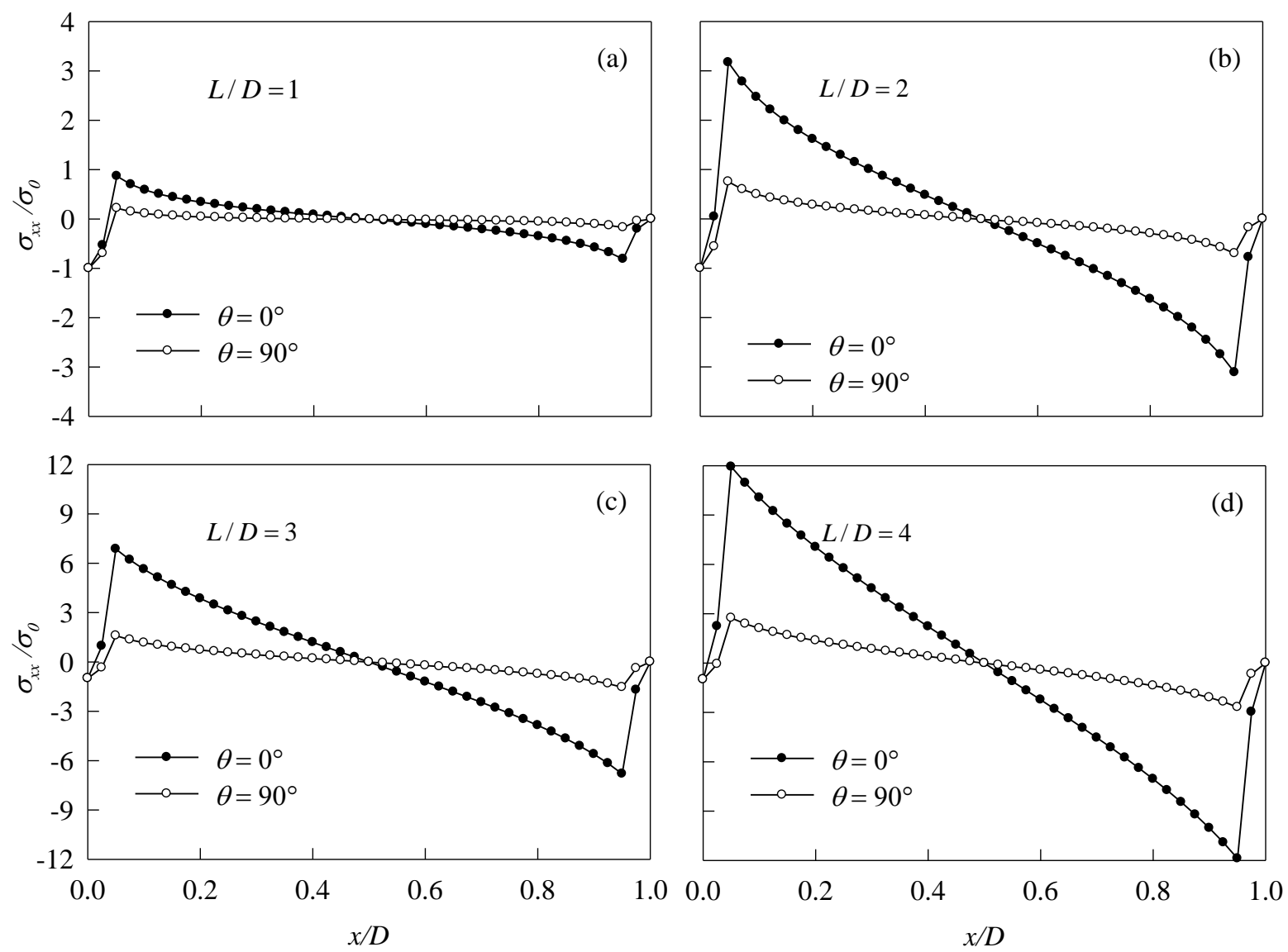

Figure 11. Distribution of lateral stress at the supporting end of the cantilever beam with global support under the uniform transverse loading. 

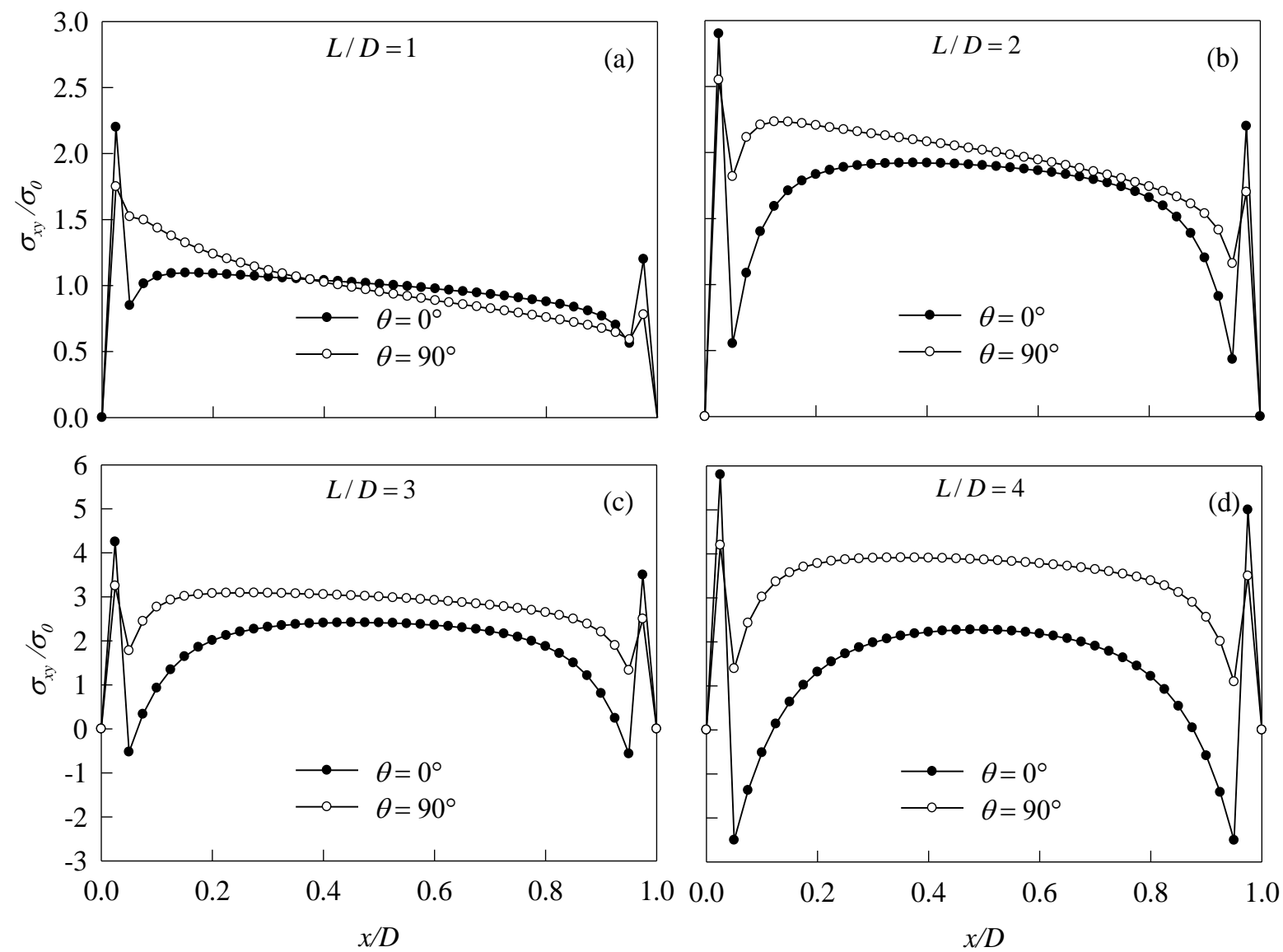

Figure 12. Distribution of shear stress at the supporting end of the cantilever beam with global support under the uniform transverse loading. 

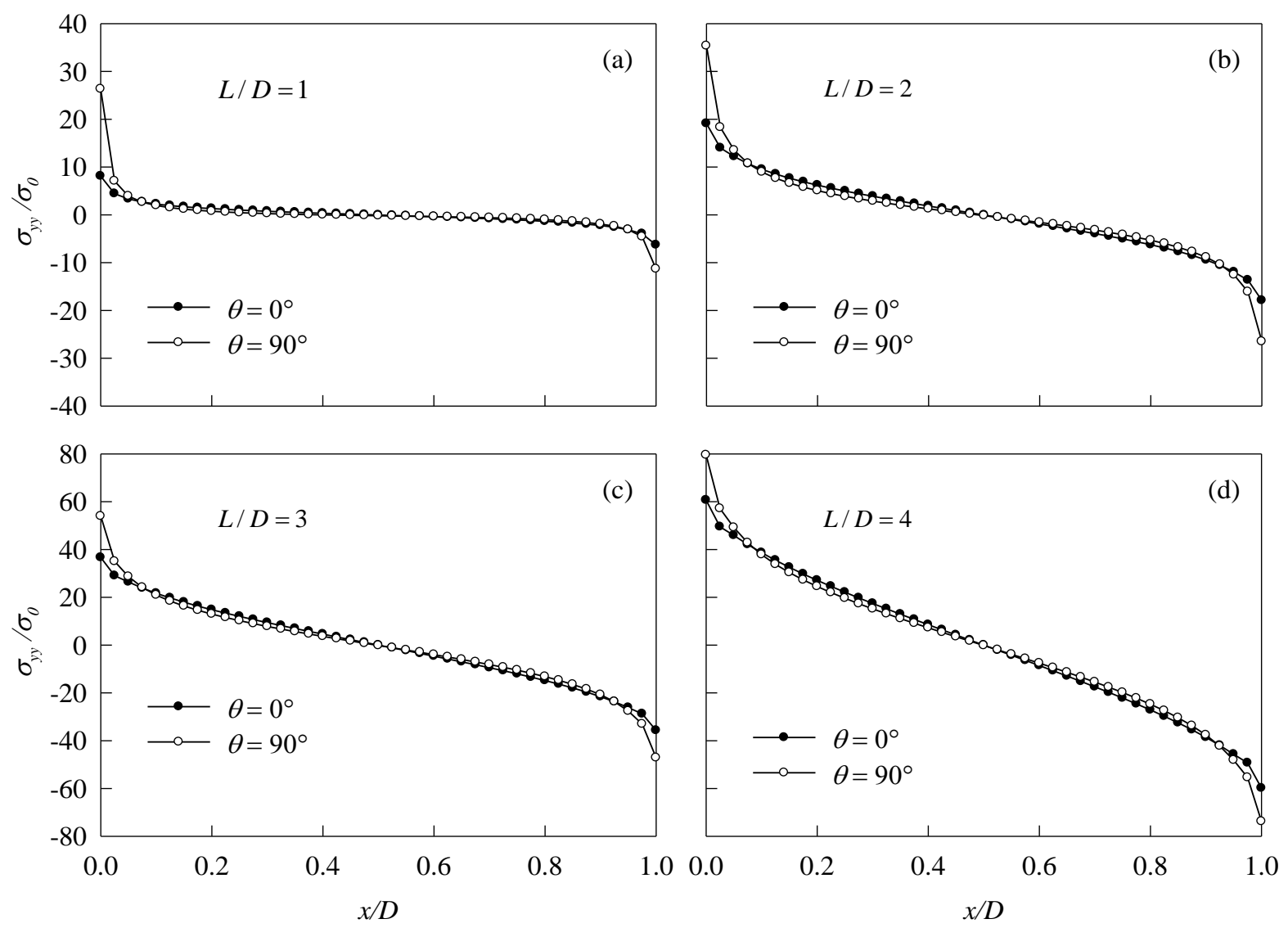

Figure 13. Distribution of bending stress at the supporting end of the cantilever beam with global support under the uniform transverse loading. 

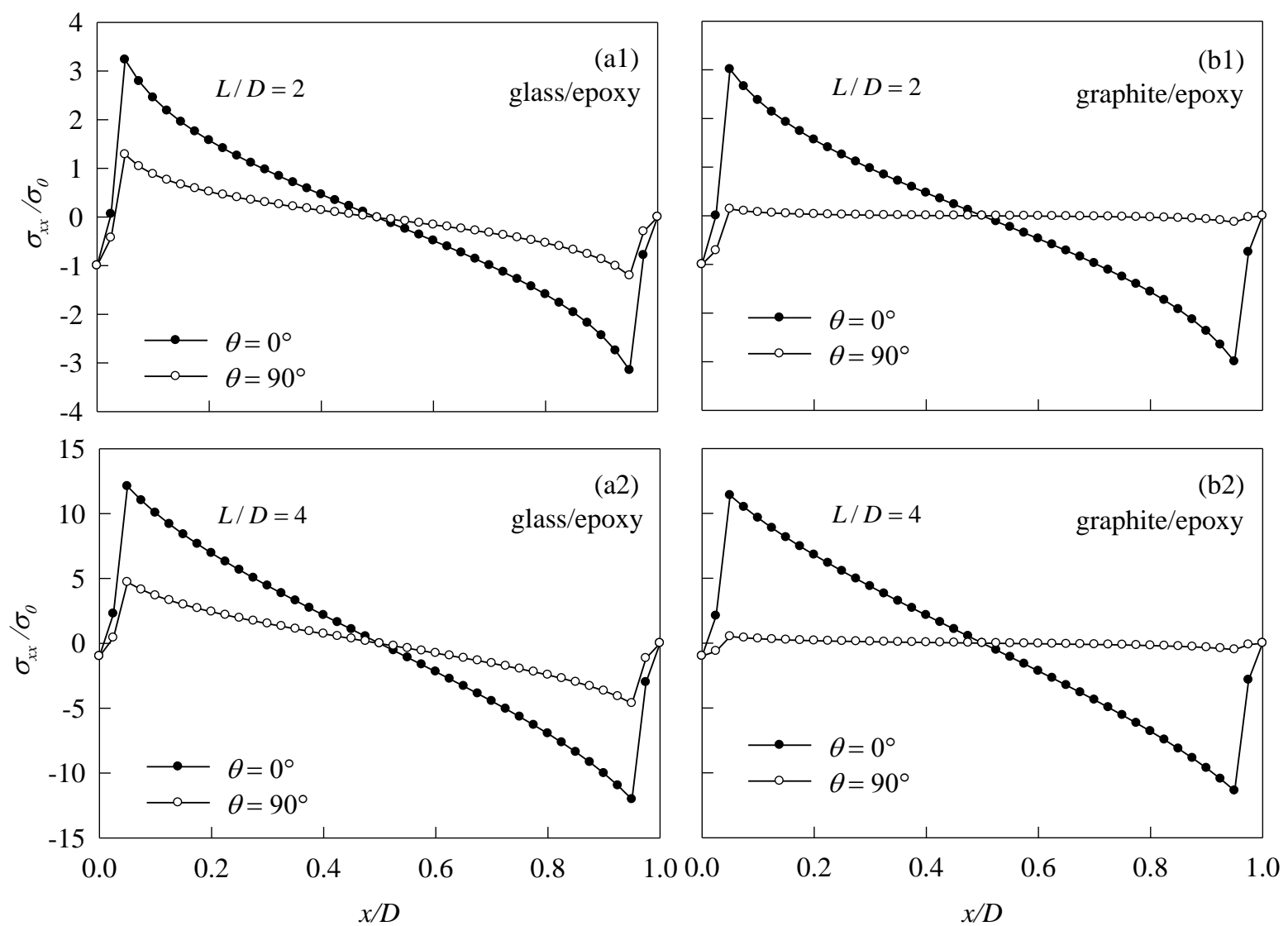

Figure 14. Comparison of fiber orientation effect on lateral stress at the supporting end of the cantilever beam with global support under the uniform transverse loading obtained for (a) glass/epoxy and (b) graphite/epoxy composite. 

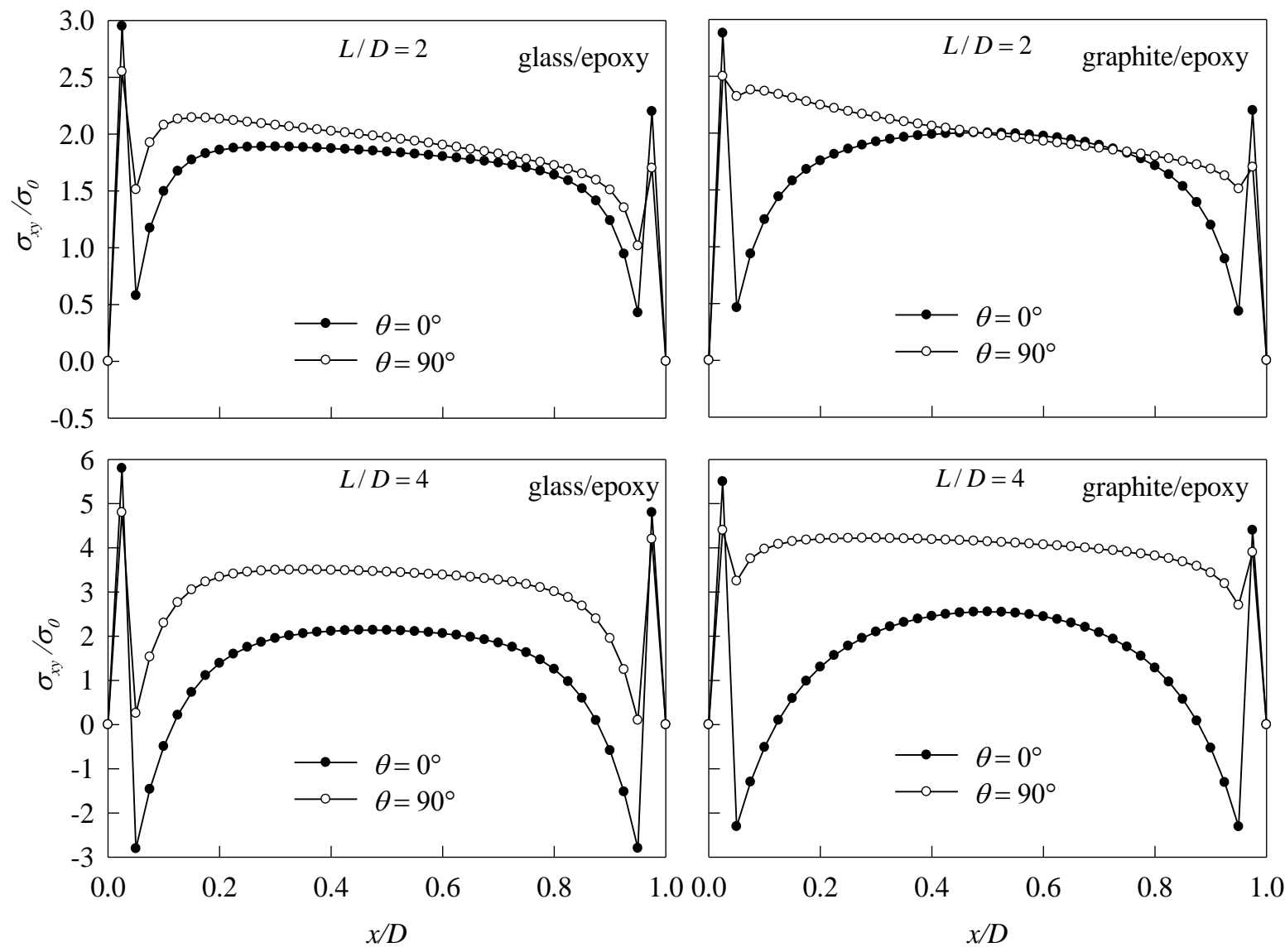

Figure 15. Comparison of fiber orientation effect on shear stress at the supporting end of the cantilever beam with global support under the uniform transverse loading obtained for (a) glass/epoxy and (b) graphite/epoxy composite. 

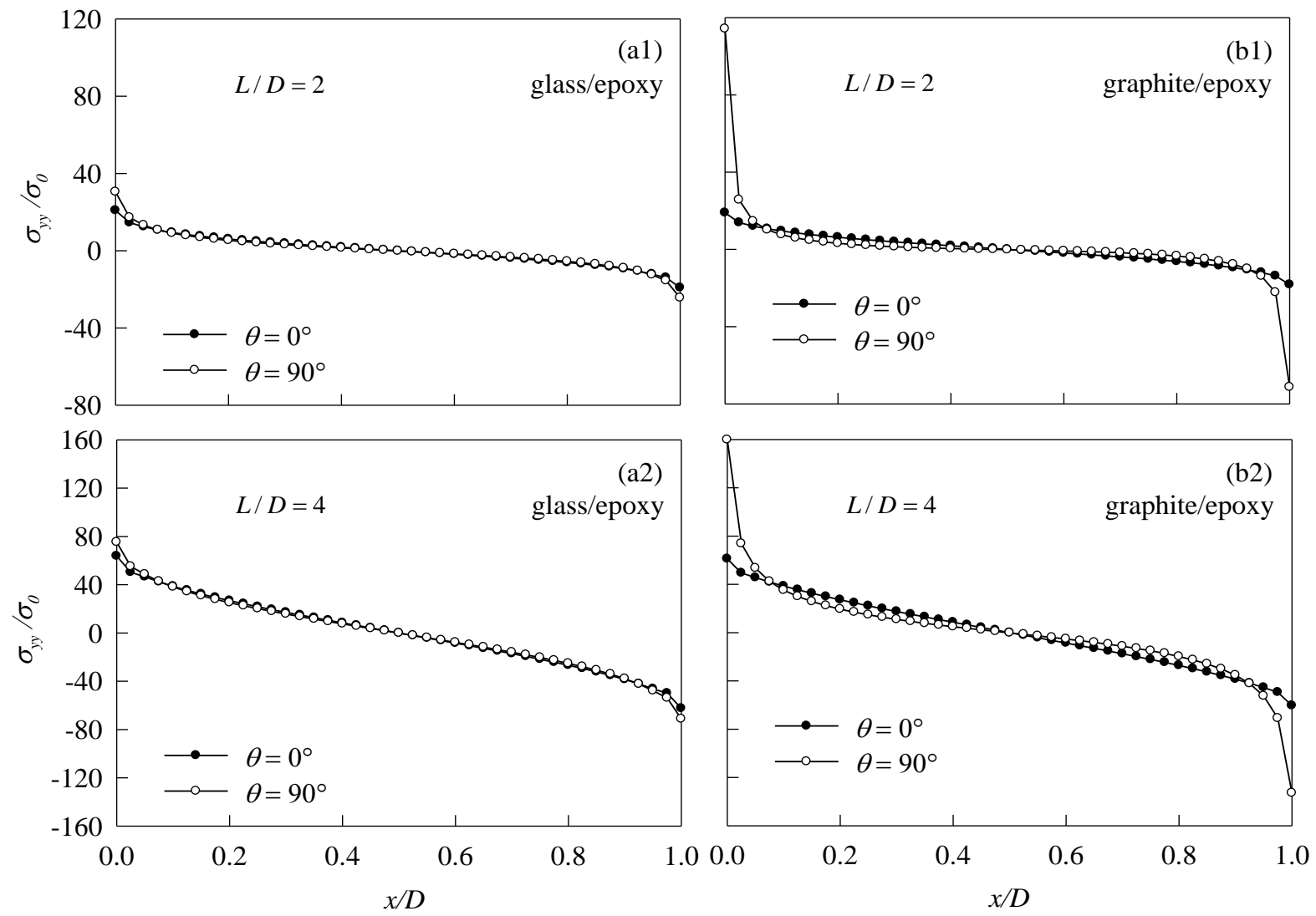

Figure 16. Comparison of fiber orientation effect on bending stress at the supporting end of the cantilever beam with global support under the uniform transverse loading obtained for (a) glass/epoxy and (b) graphite/epoxy composite. 

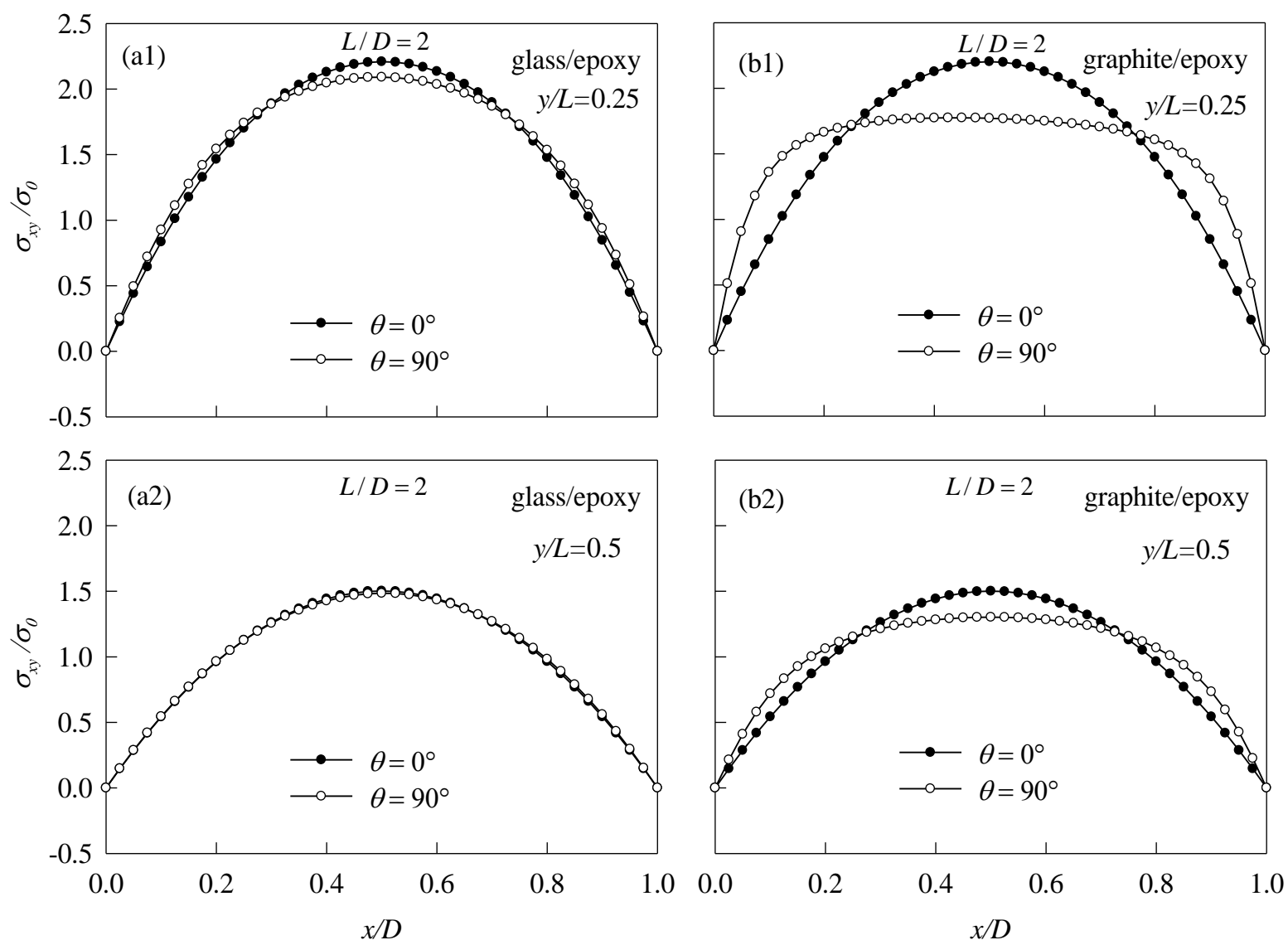

Figure 17. Comparison of fiber orientation effect on shear stress at sections away from the supporting end of the cantilever beam with global support under the uniform transverse loading obtained for (a) glass/epoxy and (b) graphite/epoxy composite. 

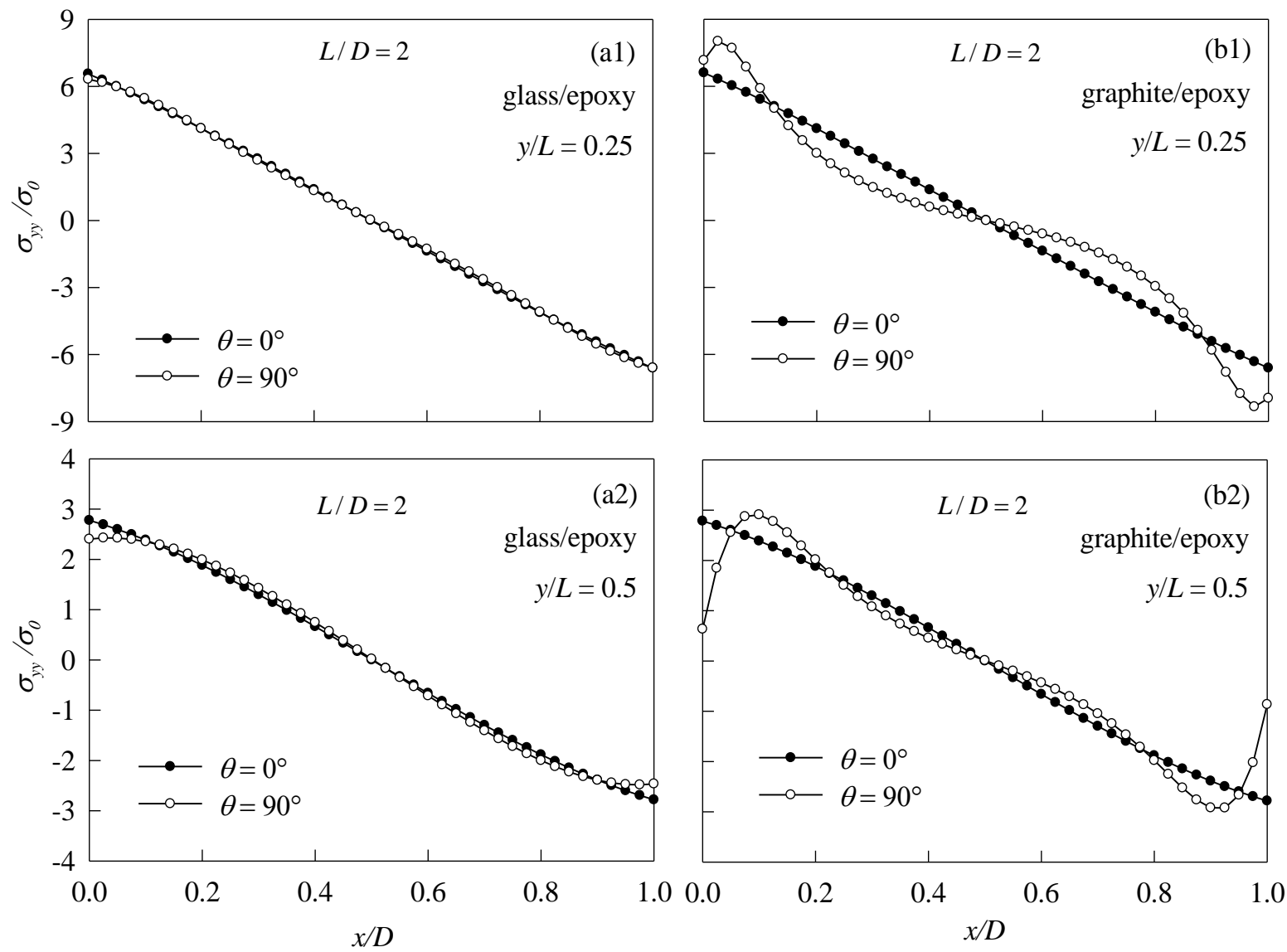

Figure 18. Comparison of fiber orientation effect on bending stress at sections away from the supporting end of the cantilever beam with global support under the uniform transverse loading obtained for (a) glass/epoxy and (b) graphite/epoxy composite. 

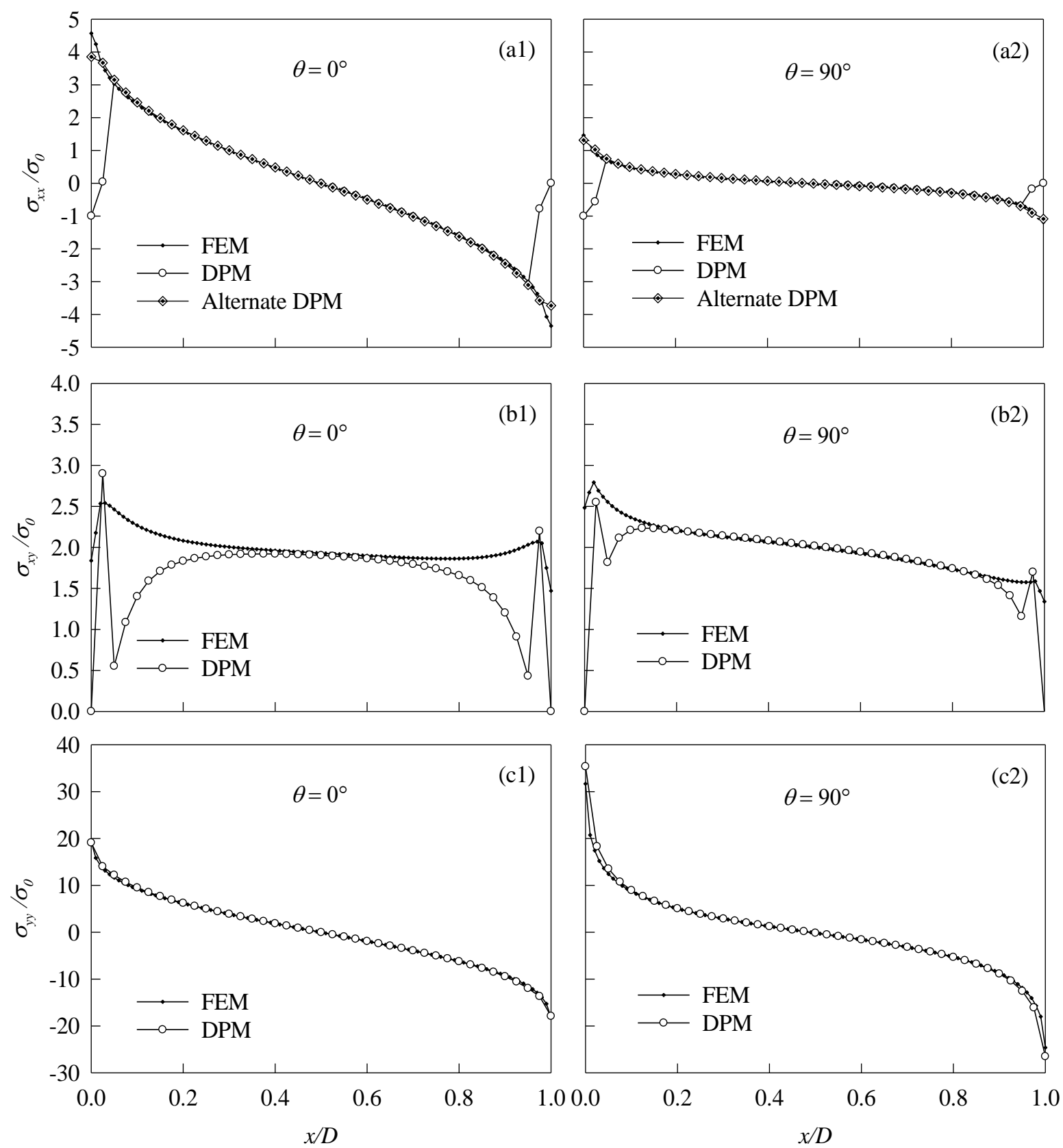

Figure 19. Comparison of different stress components: (a) lateral stress, (b) shear stress and (c) bending stress at the supporting end of a cantilever beam under the uniform transverse loading obtained by Displacement Potential and FE methods for aspect ratio of $L / D=2$. 